\title{
Solvability analysis and numerical approximation of linearized cardiac electromechanics
}

\author{
Boris Andreianov \\ Laboratoire de Mathématiques CNRS UMR 6623, \\ Département de Mathématiques, Université de Franche-Comté, \\ 16 Route de Gray, 25030 Besançon Cedex, France \\ Laboratoire Jacques-Louis Lions CNRS UMR 7598, \\ Université Pierre et Marie Curie, \\ 75252 Paris, France \\ boris.andreianov@univ-fcomte.fr \\ Mostafa Bendahmane* \\ Institut de Mathématiques de Bordeaux UMR CNRS 5251, \\ Université Victor Segalen Bordeaux 2, \\ F-33076 Bordeaux Cedex, France \\ mostafa.bendahmane@u-bordeaux2.fr \\ Alfio Quarteroni ${ }^{\dagger}$ \\ Modeling and Scientific Computing CMCS-MATHICSE-SB, \\ École Polytechnique Fédérale de Lausanne EPFL, \\ CH-1015 Lausanne, Switzerland \\ alfio.quarteroni@epfl.ch \\ Ricardo Ruiz-Baier \\ Institut des Sciences de la Terre, \\ Faculté des Géosciences et de l'Environnement, \\ Géopolis UNIL-Mouline, Université de Lausanne, \\ CH-1015 Lausanne, Switzerland \\ ricardo.ruizbaier@unil.ch \\ Received 31 March 2014 \\ Revised 16 September 2014 \\ Accepted 2 October 2014 \\ Published 9 December 2014 \\ Communicated by F. Brezzi
}

* Corresponding author

†On leave from MOX — Modellistica e Calcolo Scientifico, Dipartimento di Matematica "F. Brioschi", Politecnico di Milano, via Bonardi 9, 20133 Milano, Italy. 
This paper is concerned with the mathematical analysis of a coupled elliptic-parabolic system modeling the interaction between the propagation of electric potential and subsequent deformation of the cardiac tissue. The problem consists in a reactiondiffusion system governing the dynamics of ionic quantities, intra- and extra-cellular potentials, and the linearized elasticity equations are adopted to describe the motion of an incompressible material. The coupling between muscle contraction, biochemical reactions and electric activity is introduced with a so-called active strain decomposition framework, where the material gradient of deformation is split into an active (electrophysiology-dependent) part and an elastic (passive) one. Under the assumption of linearized elastic behavior and a truncation of the updated nonlinear diffusivities, we prove existence of weak solutions to the underlying coupled reactiondiffusion system and uniqueness of regular solutions. The proof of existence is based on a combination of parabolic regularization, the Faedo-Galerkin method, and the monotonicity-compactness method of Lions. A finite element formulation is also introduced, for which we establish existence of discrete solutions and show convergence to a weak solution of the original problem. We close with a numerical example illustrating the convergence of the method and some features of the model.

Keywords: Electromechanical coupling; bidomain equations; active deformation; weak solutions; weak compactness method; weak-strong uniqueness; finite element approximation; convergence of approximations.

AMS Subject Classification: 74F99, 35K57, 92C10, 65M60

\section{Introduction}

We are interested in the mathematical study of the interaction between the propagation of the electrical potential through the cardiac tissue, on the one side, and the related elastic mechanical response, on the other side. These distinctive processes in the cardiac function are intimately connected by several complex processes taking place at different spatio-temporal scales. In terms of macroscopic continuummechanics, the propagation of the electrical potential through the heart can be described by the so-called bidomain equations (see e.g. Ref. 45). These are obtained by writing the conservation of electrical fluxes between the extra- and intra-cellular domains separated by a membrane acting as a capacitor. Within these domains, the conductivities are of different magnitudes, and they also change depending on the particular orientation of the cardiac tissue fibers. Homogenization arguments yield a multicontinuum description of the heart where at each material point both constituents (intra- and extra-cellular material) coexist. Still at the macroscopic level, muscle deformation can be described by the equations of motion for a hyperelastic material, written in the reference configuration. The medium itself is active, in the sense that it is able to contract without the need of external loads, but rather influenced by intrinsic mechanisms taking place essentially at the microscale. In order to incorporate these effects one can follow different approaches. For instance, it is commonly assumed that stresses are additively decomposed into active and passive parts, leading to the so-called active stress formulation (see applications of such a formalism in e.g. Refs. 11, 19, 24, 31, 32 and 47). Alternatively, one can adopt the active strain formulation, ${ }^{13,30}$ for which a factorization of the deformation gradient into an active and a passive factors is assumed. Such a decomposition 
implies, in particular, that the fiber contraction driven by the depolarization of the cardiomyocytes rewrites in the mechanical balance of forces as a prescribed active deformation, rather than as an additive contribution to the stress. Moreover, this approach directly incorporates the micro-level information on the fiber contraction and fiber directions in the kinematics (through the active part of the deformation gradient), without the intermediate transcription of their role in terms of stress. ${ }^{3}$ These mechanisms essentially translate into a dependence of the strain energy function on auxiliary internal state variables, which represent the level of mechanical tissue activation passed across scales. ${ }^{39}$ Here we follow the latter alternative, but we refer to Refs. 4 and 40 for comparisons between the two approaches in terms of numerical implementation, constitutive issues, and stability.

The mathematical analysis of macroscopic cardiac models has been mainly related with the study of solutions to the bidomain equations coupled with phenomenological or physiologically-based ionic models. A variational approach was first introduced in Ref. 15 and later extended in different directions including degeneration of conductivity tensors, ${ }^{8}$ the coupling with the electrical conduction in the torso, ${ }^{11,45}$ incorporation of the specialized fast conduction system, ${ }^{10}$ analyzing the coupled system using a semigroup approach, ${ }^{12}$ deriving global classical solutions, ${ }^{27}$ and including more involved ionic models. ${ }^{49}$ On the other hand, existence theorems of general nonlinear elasticity can be found in Refs. 14 and 6, whereas applications of those theories to the particular case of hyperelastic materials and cardiac mechanics and their discretizations include e.g. Refs. 31, 22, 20, 40, 46 and 7. However, even if the literature related to numerical methods and models for cardiac electromechanics is quite large (see, for instance, Refs. 19, 24, 32, 34 and 47), rigorous studies about solvability and stability of solutions are still not well established. To the authors' knowledge, the only available existence results devoted specifically to cardiac electromechanics correspond to those by Pathmanathan et al. ${ }^{35,36}$ who analyzed a general model where the activation depends on the local stretch rate, and derived constraints on the initial data. In this work we also assume linearized elasticity equations, but we follow a different activation model (our active-strainbased description depends only on ionic quantities and we do not consider viscous effects) and employ the bidomain equations coupled with a different ionic model.

In this paper the electromechanical coupling is achieved by considering that the evolution of the electrical potentials governed by the bidomain equations depend on the deformation gradient, which enters into the bidomain equations after a transformation of coordinates from Eulerian to Lagrangian, and by virtue of the Piola identity. The coupling in the opposite direction is modeled by assuming that the active part of the deformation incorporates the influence of the calcium kinetics (or in its absence, a calcium-like scalar field) into the balance equations for the structural mechanics, which are here restricted to the linear regime.

We establish existence of weak solutions to a simplified version of the fully coupled cardiac electromechanical problem by means of the Faedo-Galerkin method combined with compactness arguments. The fully discrete counterpart of the 
proposed simplified system consists in a finite element family of piecewise quadratic elements for the approximation of deformations and piecewise linear approximations of solid pressure, electric potentials, and activation field (local strain), whereas a first-order backward Euler method is applied for the discretization in time. A linearized version of the coupled system is introduced to analyze the numerical scheme, however such a linearization turns the convergence proof more delicately than in the continuous case (see Secs. 3 and 5). We also prove a uniqueness result for the continuous problem in a weak-strong comparison setting. While some classical estimates and arguments are sketched, we concentrate on the main analytical aspects of the proofs. In the remainder of our presentation, modeling, numerical and implementation details will be reduced as much as possible, and the interested reader is referred to Refs. 33, 40 and 39 for further specifications and related models with higher complexity. We stress that our goal is to set a baseline theoretical framework for the study of more complex coupled multiphysics cardiac problems, and we believe that the present simplified electromechanical system can already exhibit some key resemblances with more involved and physiologically relevant models.

We have organized the contents of this paper as follows. Section 2 collects the main aspects of the cardiac electromechanical model we analyze, presenting the equations of passive nonlinear mechanics, the bidomain system, and the activestrain-based coupling strategy. We also list the basic assumptions of the model and provide a definition of weak solution. In Sec. 3 we state and prove the solvability of the continuous problem employing Galerkin approximations and classical compactness theory. Then, in Sec. 4 we develop a strong-weak uniqueness argument and briefly discuss regularity of solutions needed to apply it. The fully discrete linearized finite element formulation, along with additional analytic arguments developed for the proof of convergence, and two numerical tests are presented in Sec. 5. We close with some remarks and discussion of future directions in Sec. 6.

\section{Governing Equations for the Electromechanical Coupling}

\subsection{A general nonlinear elasticity problem}

Let us consider a homogeneous continuous material occupying in the initial undeformed configuration a bounded domain $\Omega \subset \mathbb{R}^{d}(d=3)$ with Lipschitz continuous boundary $\partial \Omega$. We look for the deformation field $\boldsymbol{u}: \Omega \rightarrow \mathbb{R}^{d}$ that maps a material particle that originally occupied the position $\boldsymbol{x}$ to its current position $\hat{\boldsymbol{x}}(\boldsymbol{x})=\boldsymbol{u}$. The tensor gradient of deformation is $\mathbf{D} \boldsymbol{u}$, where $\mathbf{D}$ denotes the gradient operator with respect to the material coordinates $\boldsymbol{x}$. The cardiac tissue is assumed hyperelastic, and there exists a strain stored energy function $\mathcal{W}=\mathcal{W}(\mathbf{D} \boldsymbol{u})$ from which constitutive relations between strain and stresses are obtained. We further assume incompressibility of the material, that is, the total elastic energy is minimized subject to the usual local constraint $\operatorname{det}(\mathbf{D} \boldsymbol{u})=1$, which is enforced via a scalar Lagrange multiplier $p$, interpreted as pressure. As a measure of stresses we 
use the first Piola-Kirchhoff tensor obtained from $\mathcal{W}$ by direct differentiation:

$$
\mathbf{P}=\frac{\partial \mathcal{W}}{\partial(\mathbf{D} \boldsymbol{u})}-p \operatorname{Cof}(\mathbf{D} \boldsymbol{u})
$$

where $\operatorname{Cof}(\cdot)$ is the cofactor matrix. The balance equations for deformations and pressure read as: find $\boldsymbol{u}, p$ such that:

$$
\begin{aligned}
\nabla \cdot \mathbf{P}(\mathbf{D} \boldsymbol{u}, p)+\boldsymbol{f}=\mathbf{0} & \text { in } \Omega, \\
\operatorname{det}(\mathbf{D} \boldsymbol{u})=1 & \text { in } \Omega,
\end{aligned}
$$

completed with the Robin boundary data

$$
\mathbf{P} \boldsymbol{n}=\alpha \boldsymbol{u} \text { on } \partial \Omega,
$$

where $\boldsymbol{f}$ is a prescribed body force, $\boldsymbol{n}$ stands for the unit outward normal vector to $\partial \Omega$, and $\alpha>0$ is a constant parameter. Boundary data as $(2.2)$ can be tuned to mimic the global motion of the cardiac muscle, ${ }^{39}$ without resorting to unphysiological boundary treatment typically found in the literature, as excessively rigid boundary conditions, or fixing the atrioventricular plane, or leaving the tissue completely free to move. Evidently, the precise form of the first equation in (2.1) depends on the particular constitutive relation defining $\mathcal{W}$. For sake of clarity we restrict ourselves to the case of neo-Hookean materials, that is, $\mathcal{W}=\frac{1}{2} \mu \operatorname{tr}\left[(\mathbf{D} \boldsymbol{u})^{2}-\mathbf{I}\right]$, which gives $\mathbf{P}=\mu \mathbf{D} \boldsymbol{u}-p \operatorname{Cof}(\mathbf{D} \boldsymbol{u})$, where $\mu$ is an elastic modulus. Even if simplified, such a description of the passive response of the muscle already features a nonlinear strain-stress relationship arising from the incompressibility constraint and, as will be discussed later on, anisotropy inherited from the active strain incorporation. More involved models can be found in e.g. Refs. 31, 40 and 39.

\subsection{The bidomain equations}

The description of the electrophysiology in the cardiac tissue is incorporated in the model in the form of the so-called bidomain equations. ${ }^{48}$ The quantities of interest are the intra- and extra-cellular electric potentials $\left(v_{i}=v_{i}(\boldsymbol{x}, t), v_{e}=v_{e}(\boldsymbol{x}, t)\right.$ respectively), the transmembrane potential $v=v(\boldsymbol{x}, t):=v_{i}-v_{e}$, and the so-called gating or recovery variable $w=w(\boldsymbol{x}, t)$ at $(\boldsymbol{x}, t) \in \Omega_{T}:=\Omega \times(0, T)$, where $T$ is the final time instant. The electrical conductivity of the tissue is represented by the orthotropic tensors

$$
\mathbf{K}_{k}(\boldsymbol{x})=\sigma_{k}^{l} \boldsymbol{d}_{l} \otimes \boldsymbol{d}_{l}+\sigma_{k}^{t} \boldsymbol{d}_{t} \otimes \boldsymbol{d}_{t}+\sigma_{k}^{n} \boldsymbol{d}_{n} \otimes \boldsymbol{d}_{n}, \quad k \in\{e, i\},
$$

where $\sigma_{k}^{s}=\sigma_{k}^{s}(\boldsymbol{x}) \in \boldsymbol{C}^{1}\left(\mathbb{R}^{3}\right), k \in\{e, i\}, s \in\{l, t, n\}$, are the intra- and extra-cellular conductivities along, transversal, and normal to the direction of the fibers, respectively. The fibers direction being a local quantity, we have $\boldsymbol{d}_{s}=\boldsymbol{d}_{s}(\boldsymbol{x}), s \in\{l, t, n\}$. The stimulation current externally applied to the intra- and extra-cellular spaces is represented by the functions $I_{\text {app }}^{i}, I_{\text {app }}^{e}$. The system corresponds to

$$
\chi c_{\mathrm{m}} \partial_{t} v-\nabla \cdot\left(\mathbf{K}_{\mathrm{i}} \nabla v_{i}\right)+\chi I_{\text {ion }}(v, w)=I_{\text {app }}^{i},
$$




$$
\begin{aligned}
\chi c_{\mathrm{m}} \partial_{t} v+\nabla \cdot\left(\mathbf{K}_{\mathrm{e}} \nabla v_{e}\right)+\chi I_{\mathrm{ion}}(v, w) & =I_{\mathrm{app}}^{e}, \\
\partial_{t} w-H(v, w) & =0, \quad(\boldsymbol{x}, t) \in \Omega_{T},
\end{aligned}
$$

where we recall that $v=v_{i}-v_{e}$. Here $c_{\mathrm{m}}$ and $\chi$ are model parameters. Problem (2.3) is provided with homogeneous Neumann boundary conditions for all fields. The choice of the membrane model to be used is reflected in the functions $H(v, w)$ and $I_{\text {ion }}(v, w)$. For a phenomenological description of the action potential, it suffices to consider the FitzHugh-Nagumo model, ${ }^{18,29}$ given as in assumption (E.6) below.

\subsection{The active strain model for the coupling of elasticity and bidomain equations}

In the so-called active strain model for cardiac modeling, ${ }^{13}$ the deformation gradient $\mathbf{D} \boldsymbol{u}$ is factorized into a passive part acting at a macroscale (tissue level), and an active factor operating at the microscale (cellular level), $\mathbf{D} \boldsymbol{u}=\mathbf{F}_{p} \mathbf{F}_{a}$. This implies that an intermediate configuration exists between the reference and the current frames. In that configuration, we rewrite the strain energy in such a way that the stress tensor is given by

$$
\mathbf{P}=\mu \mathbf{D} \boldsymbol{u} \mathbf{C}_{a}^{-1}-p \operatorname{Cof}(\mathbf{D} \boldsymbol{u}),
$$

where $\mathbf{C}_{a}^{-1}:=\operatorname{det}\left(\mathbf{F}_{a}\right) \mathbf{F}_{a}^{-1} \mathbf{F}_{a}^{-T}$ (see also Refs. 3 and 33). In order to cover the electrical-to-mechanical coupling, the active deformation is assumed to depend directly on the electrophysiology through the relation

$$
\mathbf{F}_{a}=\mathbf{I}+\gamma_{l} \boldsymbol{d}_{l} \otimes \boldsymbol{d}_{l}+\gamma_{t} \boldsymbol{d}_{t} \otimes \boldsymbol{d}_{t}+\gamma_{n} \boldsymbol{d}_{n} \otimes \boldsymbol{d}_{n},
$$

where for $s=l, t, n, \gamma_{s}$ are quantities whose evolution depends, nonlocally in time, on the electrophysiology equations. Notice that the onset of mechanical activation is mainly influenced by intra-cellular calcium release, ${ }^{34,36,45}$ and in particular, the dynamics of local strain follow closely those of calcium release rather than those from the transmembrane potential, as reported in Ref. 9. In the absence of calcium concentration in the FitzHugh-Nagumo model, the aforementioned fact suggests that it is $w$ (the slow wave) that better approximates the spatio-temporal structure of calcium. More physiologically involved activation models require a dependence of $\gamma_{s}$ not only on calcium, but also on local stretch, local stretch rate, sliding velocity of crossbridges, and on other force-length experimental relations, ${ }^{36,39,43}$ but for sake of simplicity we restrict ourselves to a phenomenological description of local activation in terms of the gating variable $w$.

The scalar fields $\gamma_{l}, \gamma_{t}$ and $\gamma_{n}$ can be written as functions of a parameter $\gamma$ :

$$
\gamma_{l, t, n}=\gamma_{l, t, n}(\gamma)
$$

where $\gamma_{l, t, n}: \mathbb{R} \mapsto\left[-\Gamma_{l, t, n}, 0\right]$ are Lipschitz continuous monotone functions. The values $\Gamma_{l, t, n}$ should be small enough, so that to ensure that $\operatorname{det} \mathbf{F}_{a}$ stays uniformly 
far from zero, for $\gamma \in \mathbb{R}$. The scalar field $\gamma$ is the solution of the following ODE associated to the solution $\left(v_{i}, v_{e}, w\right)$ of the bidomain system $(2.3)$ :

$$
\partial_{t} \gamma-G(\gamma, w)=0, \quad(\boldsymbol{x}, t) \in \Omega_{T},
$$

where $G(\gamma, w)=\beta\left(\eta_{1} w-\eta_{2} \gamma\right)$, for positive parameters $\beta, \eta_{1}, \eta_{2}$ (see Ref. 42 ). It remains to fix the form of the functions $\gamma_{l, t, n}$, which we assume to be

$$
\gamma_{l, t, n}=-\Gamma_{l, t, n} \frac{2}{\pi} \arctan \left(\gamma^{+} / \gamma_{R}\right), \quad \text { where } \gamma_{R} \text { is a reference value. }
$$

The form of the active strain and (2.4) yields the following expression for the total stress (where a "purely passive" and an "active" part of the stress can be readily identified),

$$
\mathbf{P}=\mu \mathbf{D} \boldsymbol{u}-p \operatorname{Cof}(\mathbf{D} \boldsymbol{u})-\mu \sum_{s \in\{l, t, n\}} \frac{\gamma_{s}\left(\gamma_{s}+2\right)}{\left(1+\gamma_{s}\right)^{2}} \boldsymbol{d}_{s} \otimes \boldsymbol{d}_{s}
$$

After assuming transverse isotropy (which translates into $\gamma_{t}=\gamma_{n}$ ) and incompressibility at the fiber level (i.e. $\operatorname{det} \mathbf{F}_{a}=1$ ), the previous expression reduces to

$$
\mathbf{P}=\mu \mathbf{D} \boldsymbol{u}-p \operatorname{Cof}(\mathbf{D} \boldsymbol{u})+\mu \gamma \mathbf{D} \boldsymbol{u}-\mu \gamma_{l}\left(1+\frac{\gamma_{l}+2}{\left(1+\gamma_{l}\right)^{2}}\right) \boldsymbol{d}_{l} \otimes \boldsymbol{d}_{l} .
$$

Details can be found in e.g. Refs. 23 and 43.

The mechanical-to-electrical coupling is achieved by pulling back the bidomain equations to the reference configuration, which, by virtue of the Piola identity, leads to a conduction term depending on the deformation gradient $\mathbf{D} \boldsymbol{u}$.

Summarizing, the active strain formulation for the electromechanical activity in the heart is written as follows ${ }^{33}$ :

$$
\begin{aligned}
-\nabla \cdot(a(\boldsymbol{x}, \gamma, \mathbf{D} \boldsymbol{u}, p)) & =\boldsymbol{f} \quad \text { in } \Omega, \\
\chi c_{\mathrm{m}} \partial_{t} v-\nabla \cdot\left(\mathbf{M}_{e}(\mathbf{D} \boldsymbol{u}) \nabla v_{e}\right)+\chi I_{\mathrm{ion}} & =I_{\mathrm{app}}^{e} \quad \text { in } \Omega_{T}, \\
\chi c_{\mathrm{m}} \partial_{t} v+\nabla \cdot\left(\mathbf{M}_{i}(\mathbf{D} \boldsymbol{u}) \nabla v_{i}\right)+\chi I_{\mathrm{ion}} & =I_{\text {app }}^{i} \quad \text { in } \Omega_{T}, \\
v_{i}-v_{e} & =v \quad \text { in } \Omega_{T}, \\
\partial_{t} w-H(v, w) & =0 \quad \text { in } \Omega_{T}, \\
\partial_{t} \gamma-G(\gamma, w) & =0 \quad \text { in } \Omega_{T} .
\end{aligned}
$$

Here, according to the above discussion, we should take

$$
a(\boldsymbol{x}, \gamma, \mathbf{D} \boldsymbol{u}, p):=\mu \mathbf{D} \boldsymbol{u} \mathbf{C}_{a}^{-1}(\boldsymbol{x}, \gamma)-p \operatorname{Cof}(\mathbf{D} \boldsymbol{u}),
$$

and

$$
\mathbf{M}_{k}(\mathbf{D} \boldsymbol{u}):=(\mathbf{D} \boldsymbol{u})^{-1} \mathbf{K}_{k}(\mathbf{D} \boldsymbol{u})^{-T}, \quad k \in\{i, e\} .
$$

Moreover, we have the incompressibility constraint under the form

$$
\operatorname{det}(\mathbf{D} \boldsymbol{u})=1 \quad \text { in } \Omega, \text { for a.e. } t \in(0, T) \text {. }
$$

The system (2.5) has to be completed with suitable initial data for $v, w, \gamma$ and with boundary data on $v_{i, e}$ and on the elastic flux $a(\cdot, \cdot, \cdot, \cdot)$. 


\subsection{Linearizing the elasticity equations}

In the remaining part of this paper, for the sake of simplicity of both numerical and mathematical analysis of the problem we introduce two modifications into (2.5), (2.7), (2.8). Firstly, noting that $\partial_{\mathbf{D} \boldsymbol{u}} \operatorname{det}(\mathbf{D} \boldsymbol{u})=\operatorname{det} \mathbf{D} \boldsymbol{u} \nabla \cdot \boldsymbol{u}$, we can incorporate a linearized incompressibility constraint by imposing

$$
\nabla \cdot \boldsymbol{u}=0 \text { in } \Omega \text {, for a.e. } t \in(0, T),
$$

we also linearize the flux in (2.6) with respect to $\mathbf{D} \boldsymbol{u}$ by replacing it with

$$
a(\boldsymbol{x}, \gamma, \mathbf{D} \boldsymbol{u}, p):=\mu \mathbf{D} \boldsymbol{u} \mathbf{C}_{a}^{-1}(\boldsymbol{x}, \gamma)-p \mathbf{I} .
$$

Introducing the notation $\sigma(\boldsymbol{x}, \gamma)$ for $\mu \mathbf{C}_{a}^{-1}(\boldsymbol{x}, \gamma)$, we rewrite the first equation of $(2.5)$ as

$$
-\nabla \cdot(\sigma(\boldsymbol{x}, \gamma) \mathbf{D} \boldsymbol{u})+\nabla p=\boldsymbol{f} .
$$

The linearization results, however, in the fact that the matrices $\mathbf{M}_{k}, k=i, e$ in (2.7) may become ill-defined, since the linearized incompressibility constraint does not guarantee invertibility of $\mathbf{D} \boldsymbol{u}$. We proceed to a linearization and to a truncation, so that to ensure the boundedness and coercivity (see (E.3) below) of $\mathbf{M}_{i}, \mathbf{M}_{e}$ :

$$
\begin{aligned}
\mathbf{M}_{k}(\mathbf{D} \boldsymbol{u}) & :=\mathbf{K}_{k}-T_{\delta}\left(\Delta_{k}(\mathbf{D} \boldsymbol{u})+\Delta_{k}(\mathbf{D} \boldsymbol{u})^{T}\right), \\
\text { where } \quad \Delta_{k}(\mathbf{D} \boldsymbol{u}) & :=(\mathbf{D} \boldsymbol{u}-\mathbf{I}) \mathbf{K}_{k}, \quad k \in\{i, e\},
\end{aligned}
$$

and $T_{\delta}$ is a suitable truncation function that coincides with the identity map in a neighborhood of the origin. For the sake of being definite, we set

$$
T_{\delta}(\mathbf{M}):= \begin{cases}\mathbf{M}, & \|\mathbf{M}\| \leq \delta, \\ \mathbf{M} \frac{\delta}{\|\mathbf{M}\|}, & \|\mathbf{M}\| \geq \delta,\end{cases}
$$

with some $\delta<1$.

Remark 2.1. The amplitude of contractions in the heart tissue is rather large, which makes the validity of the linearized model questionable, so we cannot claim that in its present form it is able to represent physiological regimes. However, we consider the subsequent analysis as a baseline for future developments including more accurate and more general models. In any case, we will briefly address the influence of the linearization and of the truncation on the solutions of (2.5) from a numerical viewpoint, in Sec. 5 .

\subsection{The problem to be solved and its weak formulation}

Let us consider the following class of problems:

$$
\begin{array}{r}
-\nabla \cdot(\sigma(\boldsymbol{x}, \gamma) \mathbf{D} \boldsymbol{u})+\nabla p=\boldsymbol{f}, \quad \nabla \cdot \boldsymbol{u}=0 \quad \text { in } \Omega, \text { for a.e. } t \in(0, T), \\
c_{m} \chi \partial_{t} v-\nabla \cdot\left(\mathbf{M}_{i}(\boldsymbol{x}, \mathbf{D} \boldsymbol{u}) \nabla v_{i}\right)+\chi I_{\mathrm{ion}}(v, w)=I_{\text {app }}^{i}(t, \boldsymbol{x}) \quad \text { in } \Omega_{T}, \\
c_{m} \chi \partial_{t} v+\nabla \cdot\left(\mathbf{M}_{e}(\boldsymbol{x}, \mathbf{D} \boldsymbol{u}) \nabla v_{e}\right)+\chi I_{\mathrm{ion}}(v, w)=I_{\mathrm{app}}^{e}(t, \boldsymbol{x}) \quad \text { in } \Omega_{T},
\end{array}
$$




$$
\begin{gathered}
v=v_{i}-v_{e} \quad \text { in } \Omega_{T}, \\
\partial_{t} w-H(v, w)=0 \quad \text { in } \Omega_{T}, \\
\partial_{t} \gamma-G(\gamma, w)=0 \quad \text { in } \Omega_{T} .
\end{gathered}
$$

Equations (2.12)-(2.14) are complemented with the boundary data (including the linearization of $(2.2))$ :

$$
\sigma(\boldsymbol{x}, \gamma) \mathbf{D} \boldsymbol{u} \boldsymbol{n}-p \boldsymbol{n}=\alpha \boldsymbol{u} \quad \text { on } \partial \Omega, \text { for a.e. } t \in(0, T),
$$

for some $\alpha>0$ and

$$
\left(\mathbf{M}_{k}(\boldsymbol{x}, \mathbf{D} \boldsymbol{u}) \nabla v_{k}\right) \cdot \boldsymbol{n}=0 \quad \text { on }(0, T) \times \partial \Omega, \quad k=i, e
$$

(different boundary conditions can be imposed on $v_{i, e}$; the choice of Neumann conditions (2.19) results in the compatibility constraint (2.23) below). The initial data are:

$$
v(0, \cdot)=v_{0}, \quad w(0, \cdot)=w_{0}, \quad \gamma(0, \cdot)=\gamma_{0} \quad \text { in } \Omega .
$$

The following properties of the model (2.12)-(2.17) and (2.18)-(2.20) are instrumental for the subsequent analysis:

(E.1) $(\sigma(\boldsymbol{x}, \gamma))_{x \in \Omega, \gamma \in \mathbb{R}}$ is a family of symmetric tensors, uniformly bounded and positive definite:

$$
\begin{gathered}
\exists c>0 \text { : for a.e. } x \in \Omega, \quad \forall \gamma \in \mathbb{R} \quad \forall \mathbf{M} \in \mathbb{M}_{3 \times 3}, \\
\qquad \frac{1}{c}|\mathbf{M}|^{2} \leq(\sigma(\boldsymbol{x}, \gamma) \mathbf{M}): \mathbf{M} \leq c|\mathbf{M}|^{2} ;
\end{gathered}
$$

(E.2) the map $\gamma \mapsto \sigma(\cdot, \gamma)$ is uniformly Lipschitz continuous;

(E.3) $\left(\mathbf{M}_{i, e}(\boldsymbol{x}, \mathbf{M})\right)_{x \in \Omega, \mathbf{M} \in \mathbb{M}_{3 \times 3}}$ is a family of symmetric matrices, uniformly bounded and positive definite:

$$
\begin{gathered}
\exists c>0 \text { : for a.e. } x \in \Omega, \quad \forall \mathbf{M} \in \mathbb{M}_{3 \times 3} \quad \forall \xi \in \mathbb{R}^{3}, \\
\qquad \frac{1}{c}|\xi|^{2} \leq\left(\mathbf{M}_{i, e}(\boldsymbol{x}, \mathbf{M}) \xi\right) \cdot \xi \leq c|\xi|^{2} ;
\end{gathered}
$$

(E.4) the maps $\mathbf{M} \mapsto \mathbf{M}_{i, e}(\cdot, \mathbf{M})$ are uniformly Lipschitz continuous;

(E.5) the function $G$ is given by $G(\gamma, w)=\eta_{1}\left(\beta w-\eta_{2} \gamma\right)$ with $\beta, \eta_{1}, \eta_{2}>0$;

(E.6) the functions $H$ and $I_{\text {ion }}$ are given by the FitzHugh-Nagumo kinetics

$$
H(v, w)=A v-B w, \quad I_{\text {ion }}(v, w)=j(v)+C w,
$$

where $j \in C^{1}(\mathbb{R})$ and $A, B, C$ are positive parameters. Moreover, we assume that there exist constants $A_{1}, A_{2}, A_{3}>0$ such that:

$$
\begin{aligned}
& j(0)=0, \quad \frac{j\left(v_{1}\right)-j\left(v_{2}\right)}{v_{1}-v_{2}}>-A_{1}, \quad \forall v_{1} \neq v_{2}, \\
& 0<A_{2} \leq \liminf _{|v| \rightarrow \infty} \frac{j(v)}{v^{3}} \leq \limsup _{|v| \rightarrow \infty} \frac{j(v)}{v^{3}} \leq A_{3} .
\end{aligned}
$$


In particular,

$$
\mathcal{B}: v \mapsto j(v) / v+A_{1}
$$

is a non-negative function that satisfies, for $|v|$ large enough, $\frac{1}{2} A_{2}|v|^{2} \leq \mathcal{B}(v) \leq$ $2 A_{3}|v|^{2}$; and the function $v \mapsto v \mathcal{B}(v)=j(v)+A_{1} v$ is monotone increasing;

(E.7) the following condition holds

$$
\int_{\Omega} I_{\text {app }}^{i}=\int_{\Omega} I_{\text {app }}^{e} \quad \text { and } \quad \int_{\Omega} v_{e}(\boldsymbol{x}, t) d \boldsymbol{x}=0 \quad \text { for a.e. } t \in(0, T) ;
$$

(E.8) the data $v_{0}, w_{0}, \gamma_{0}$ lie in $L^{2}(\Omega)$ whereas $f \in L^{2}\left(\Omega_{T}\right)^{3}$ and $I_{\text {app }}^{i, e} \in L^{2}\left(\Omega_{T}\right)$.

Note that, in practice, one starts with an undeformed configuration, i.e. with $\gamma \equiv 0$

Observe also that the above system $(2.5)$, (2.9) with $a(\cdot, \cdot, \cdot, \cdot)$ and $\mathbf{M}_{i, e}(\cdot, \cdot)$ given by (2.10), (2.11) falls within the framework described by (2.12)-(2.20) and (E.1)-(E.8). Indeed, it is enough to check that assumptions (E.1)-(E.4) are satisfied (assumptions (E.5)-(E.8) are already enforced). Let us stress that due to the assumption (2.4), the properties (E.1), (E.2) hold. Similarly, the definition (2.11) along with the truncation $T_{\delta}$, with $\delta$ small enough with respect to the eigenvalues of matrices $\mathbf{K}_{k}$, guarantees (E.3), (E.4).

Due to the properties (E.1)-(E.8), the following weak formulation makes sense.

Definition 2.1. A weak solution of problem $(2.12)-(2.20)$ is $U=\left(\boldsymbol{u}, p, v_{i}, v_{e}, v\right.$, $w, \gamma)$ such that we have the following:

(i) $\boldsymbol{u} \in L^{2}\left(0, T ; H^{1}(\Omega)^{3}\right), p \in L^{2}\left(\Omega_{T}\right), v_{i, e} \in L^{2}\left(0, T ; H^{1}(\Omega)\right) ; v \in E:=L^{2}(0, T$; $\left.H^{1}(\Omega)\right) \cap L^{4}\left(\Omega_{T}\right)$ with $\partial_{t} v \in E^{\prime}:=L^{2}\left(0, T ;\left(H^{1}(\Omega)\right)^{\prime}\right)+L^{4 / 3}\left(\Omega_{T}\right) ;$ and $\gamma, w \in$ $C^{1}\left(0, T ; L^{2}(\Omega)\right)$.

(ii) For a.e. $t \in(0, T)$ for all $\boldsymbol{v} \in H^{1}(\Omega)^{3}$ there holds

$$
\int_{\Omega}(\sigma(\boldsymbol{x}, \gamma) \mathbf{D} \boldsymbol{u}: \mathbf{D} \boldsymbol{v}+D p \cdot \boldsymbol{v})=\int_{\Omega} \boldsymbol{f} \cdot \boldsymbol{v}+\int_{\partial \Omega} \alpha \boldsymbol{u} \cdot \boldsymbol{v}
$$

(in the last integral, $\boldsymbol{u}, \boldsymbol{v}$ are shortcuts for the traces of $\boldsymbol{u}, \boldsymbol{v}$ on $\partial \Omega$ ) and moreover $\nabla \cdot \boldsymbol{u}=0$ a.e. in $\Omega_{T}$, which can be expressed as

$$
\forall q \in H_{0}^{1}(\Omega), \quad \int_{\Omega} \boldsymbol{u} \cdot \nabla q=0 .
$$

(iii) The distributional derivative $\partial_{t} v$ can be identified with an element $b \in E^{\prime}$ such that for all $\xi \in E$ with $\xi_{t} \in L^{\infty}\left(\Omega_{T}\right)$ and $\xi(0, \cdot)=0$, there holds

$$
\begin{aligned}
& \int_{0}^{T} c_{m} \chi\langle b, \xi\rangle+\int_{0}^{T} \int_{\Omega}\left(\mathbf{M}_{i}(\boldsymbol{x}, \mathbf{D} \boldsymbol{u}) \nabla v_{i} \cdot \nabla \xi+\chi I_{\mathrm{ion}}(v, w) \xi\right)=\int_{0}^{T} \int_{\Omega} I_{\mathrm{app}}^{i} \xi \\
& \int_{0}^{T} c_{m} \chi\langle b, \xi\rangle-\int_{0}^{T} \int_{\Omega}\left(\mathbf{M}_{e}(\boldsymbol{x}, \mathbf{D} \boldsymbol{u}) \nabla v_{e} \cdot \nabla \xi+\chi I_{\mathrm{ion}}(v, w) \xi\right)=\int_{0}^{T} \int_{\Omega} I_{\mathrm{app}}^{e} \xi
\end{aligned}
$$


with

$$
\int_{0}^{T}\langle b, \xi\rangle=-\int_{0}^{T} \int_{\Omega} v \partial_{t} \xi-\int_{\Omega} v_{0} \xi(0, \cdot) ;
$$

in addition, $v=v_{i}-v_{e}$ a.e. on $\Omega_{T}$.

(iv) For a.e. $t \in(0, T)$ Eqs. (2.16), (2.17) are fulfilled in $L^{2}(\Omega)$, and $w(0, \cdot)=w_{0}$, $\gamma(0, \cdot)=\gamma_{0}$ a.e. in $\Omega$.

Here and below, following the formalism of Ref. 2, the duality product between $\xi \in E$ and $b \in E^{\prime}$ is written as $\int_{0}^{T}\langle b(t), \xi(t)\rangle d t$ where for $\xi_{0} \in H^{1}(\Omega) \cap L^{4}(\Omega)$ and $b_{0}=b_{1}+b_{2}, b_{1} \in H^{1}(\Omega)^{\prime}, b_{2} \in L^{4 / 3}(\Omega)$,

$$
\left\langle b_{0}, \xi_{0}\right\rangle:=\left(b_{1}, \xi_{0}\right)_{\left(H^{1}\right)^{\prime}, H^{1}}+\int_{\Omega} b_{2} \xi .
$$

Remark 2.2. Although the variational formulation (2.26)-(2.28) is standard in the context of degenerate parabolic problems, here we face a delicate point. Because $v_{i, e}$ do not necessarily belong to $L^{4}\left(\Omega_{T}\right)$, it is not allowed to take $v_{i}$ as test function in (2.26) nor $v_{e}$, for the test function in (2.26). Yet, thanks to the regularization approach presented in Ref. 5 [Lemma 2.3], it is possible to sum up the weak formulation (2.26) with $\xi=v_{i}$ and the weak formulation (2.26) with $\xi=v_{e}$. Indeed, our assumptions include $v \in L^{4}\left(\Omega_{T}\right)$ and $I_{\text {ion }}(v, w) \in L^{4 / 3}\left(\Omega_{T}\right)$, thus the term

$$
I_{\text {ion }}(v, w) v=I_{\text {ion }}(v, w)\left(v_{i}-v_{e}\right)=I_{\text {ion }}(v, w) v_{i}-I_{\text {ion }}(v, w) v_{e},
$$

belongs to $L^{1}\left(\Omega_{T}\right)$ even if the two terms $I_{\text {ion }}(v, w) v_{i, e}$ can be nonintegrable.

\section{Existence Proof by Convergence of Galerkin Approximations for a Regularized Problem}

In this section, we prove the main existence result of this paper and prepare the ground for proving convergence of numerical approximations of the system.

Theorem 3.1. Assume that conditions (E.1)-(E.8) hold. If $v_{0}, w_{0}, \gamma_{0} \in L^{2}(\Omega)$ and $\boldsymbol{f} \in L^{2}\left(\Omega_{T}\right)^{3}, I_{\text {app }}^{i, e} \in L^{2}\left(\Omega_{T}\right)$, then there exists a weak solution $U=\left(\boldsymbol{u}, p, v_{i}\right.$, $\left.v_{e}, v, w, \gamma\right)$ to (2.12)-(2.17) with the boundary and initial data specified as in (2.18)$(2.20)$.

Although the fixed-point techniques are also well suited for the proof of existence, here we prefer to treat it with the Galerkin method in space. A parabolic approximation similar to the one proposed in Ref. 8 is used to ensure existence of approximate solutions. The proof of convergence of the finite element approximation of Sec. 5 will follow the same guidelines, but the parabolic regularization will be replaced by the linearized time-implicit discretization. In particular, except for the $L^{4}$ bound, all the estimates that we establish in this section on the sequence of approximate solutions will also be valid for the numerical approximate solutions constructed in Sec. 5 . 


\subsection{Construction of approximate solutions}

We start by fixing some increasing, as $h \downarrow 0$, families of linear finite-dimensional subspaces $\left(\mathcal{V}^{h}\right)_{h}$ and $\left(V^{h}\right)_{h}$ of $H^{1}(\Omega)^{3}, H^{1}(\Omega)$, respectively, such that $\bigcup_{h>0} \mathcal{V}^{h}$ is dense in $H^{1}(\Omega)^{3}$ and $\bigcup_{h>0} V^{h}$ is dense both in $L^{2}(\Omega)$ and in $H^{1}(\Omega)$. In view of the structure of the FitzHugh-Nagumo nonlinearity $I_{\text {ion }}$ (see assumption (E.6)), it is convenient to ask for the inclusion $V^{h} \subset L^{4}(\Omega)$. As usual, we require the Ladyzhenskaya-Babuška-Brezzi (or inf-sup) condition

$$
\exists \beta>0: \quad \forall h>0, \quad \min _{q \in V^{h}, q \neq 0} \max _{\boldsymbol{v} \in \mathcal{V}^{h}, \boldsymbol{v} \neq 0} \frac{\int_{\Omega} q \nabla \cdot \boldsymbol{v}}{\|q\|_{L^{2}}\|\boldsymbol{v}\|_{H^{1}}} \geq \beta>0 .
$$

Such sequences of finite-dimensional subspaces do exist, see in particular Sec. 5 .

Then we look for a discrete solution $U^{h}=\left(\boldsymbol{u}^{h}, p^{h}, v_{i}^{h}, v_{e}^{h}, v^{h}, w^{h}, \gamma^{h}\right)$ with $\boldsymbol{u}^{h} \in L^{2}\left(0, T ; \mathcal{V}^{h}\right)$ and for $p^{h} \in L^{2}\left(0, T ; V^{h}\right)$ and $v_{i}^{h}, v_{e}^{h}, v^{h}, w^{h}, \gamma^{h} \in \boldsymbol{C}^{1}\left(0, T ; V^{h}\right)$ that satisfy the Galerkin formulation of (2.12)-(2.20). This means that each of the equations of the system is recast into a weak formulation on $\Omega_{T}$ where time derivatives and initial conditions (projected on $V^{h}$ by means of the $L^{2}$-Hilbertian projection $\mathbb{P}_{V^{h}}$ ) are included in a strong sense. For example, the discrete analogue of (2.26), (2.28) writes:

$$
\begin{aligned}
& v^{h}(0)=\mathbb{P}_{V^{h}}\left(v_{0}\right) \text { and } \forall \xi \in V^{h}, \\
& c_{m} \chi \frac{d}{d t} \int_{\Omega} v^{h}(t) \xi+\int_{\Omega}\left(\mathbf{M}_{i}\left(\boldsymbol{x}, \mathbf{D} \boldsymbol{u}^{h}(t)\right) \nabla v_{i}^{h}(t) \cdot \nabla \xi+\chi I_{\text {ion }}\left(v^{h}(t), w^{h}(t)\right) \xi\right) \\
& =\int_{\Omega} I_{\text {app }}^{i}(t) \xi .
\end{aligned}
$$

If necessary, $I_{\text {app }}^{i, e}$ can be regularized in time. Further, the discrete analogue of (2.24), (2.25) reads, pointwise in $t$,

$$
\begin{aligned}
& \forall \boldsymbol{v} \in \mathcal{V}^{h}, \quad \int_{\Omega} \sigma\left(\boldsymbol{x}, \gamma^{h}(t)\right) \mathbf{D} \boldsymbol{u}^{h}(t): \mathbf{D} \boldsymbol{v}+D p^{h}(t) \cdot \boldsymbol{v}=\int_{\Omega} \boldsymbol{f}(t) \cdot \boldsymbol{v}+\int_{\partial \Omega} \alpha \boldsymbol{u}^{h}(t) \cdot \boldsymbol{v}, \\
& \forall q \in V^{h}, \quad \int_{\Omega} \boldsymbol{u}^{h}(t) \cdot \nabla q=0 .
\end{aligned}
$$

Notice that after discretization, we have a system of ODEs coupled to a system of algebraic equations to be solved at every time $t$. While the ODE part of the system obeys the conditions of the Cauchy-Lipschitz theorem, because of the coupling with the algebraic part existence of a discrete solution is not obvious. To prove existence of $U^{h}$, we regularize the Galerkin discretization in the spirit of Ref. 8 . Namely, in the left-hand side of the ODE in (3.2) we add the term $\varepsilon \frac{d}{d t} \int_{\Omega} v_{i}^{h}(t) \xi$ (the term $-\varepsilon \frac{d}{d t} \int_{\Omega} v_{e}^{h}(t) \xi$ is added into the analogous equation written for $v_{e}^{h}$ ) and we add the term $\varepsilon \frac{d}{d t} \int_{\Omega} \boldsymbol{u}^{h}(t) \boldsymbol{v}$ into the left-hand side of (3.3). As for $I_{\text {app }}^{i, e}$, the source term $\boldsymbol{f}$ can be regularized in $t$. The initial data can be fixed to $\boldsymbol{u}^{h, \varepsilon}(0)=0$, $v_{i}^{\varepsilon, h}=\mathbb{P}_{V^{h}}\left(v_{0}\right) / 2, v_{e}^{\varepsilon, h}=-\mathbb{P}_{V^{h}}\left(v_{0}\right) / 2$. Then the system on the new unknown 
$U^{h, \varepsilon}$ becomes a well-posed ODE problem; solutions are defined globally on $[0, T]$ because estimates preclude finite-time explosion. Further, the estimates we establish in Sec. 3.2 below are actually valid also for $\varepsilon>0$ and moreover, they are independent of the parabolic regularization parameter $\varepsilon$. Since our functions take values into a finite-dimensional space, from these bounds it is easy to deduce strong compactness of the family $\left(v^{h, \varepsilon}, w^{h, \varepsilon}, \gamma^{h, \varepsilon}\right)_{\varepsilon}$ in $\boldsymbol{C}^{0}\left([0, T] ; V^{h}\right)^{3}$ and the weak compactness of the family $\left(v_{i}^{h, \varepsilon}, v_{e}^{h, \varepsilon}, p^{h, \varepsilon}\right)_{\varepsilon}$ in $L^{2}\left(0, T ; V^{h}\right)^{3}$ as well as the weak compactness of $\left(\boldsymbol{u}^{h, \varepsilon}\right)_{\varepsilon}$ in $L^{2}\left(0, T ; \mathcal{V}^{h}\right)$. The strong compactness of $\left(\mathbf{D} \boldsymbol{u}^{h, \varepsilon}\right)_{\varepsilon}$ needed to conclude the passage to the limit in the parabolic regularization of (3.2), as $\varepsilon \rightarrow 0$, is obtained using the Minty-Browder argument, as in Sec. 3.3 below. Thus, passing to the limit $\varepsilon \rightarrow 0$ we prove existence of $U^{h}$ solving the Galerkin discretization of problem (2.12)-(2.20).

\subsection{A priori estimates}

Assuming that there exists a solution to the above problems, we derive estimates that are uniform in $h>0$ (addition of a parabolic penalization with $\varepsilon>0$ in the equations for $\boldsymbol{u}^{h}, v_{i}^{h}, v_{e}^{h}$ leads to the same estimates, provided $\boldsymbol{u}^{h}(0), v_{i}^{h}(0), v_{e}^{h}(0)$ remain bounded).

First, according to the definition of Galerkin approximations we are authorized to take $\boldsymbol{u}^{h}$ and $p^{h}$ for the test functions in the weak formulations of the first and the second equations of (2.12), respectively. Summing up the resulting identities, we deduce that

$$
\int_{0}^{T} \int_{\Omega}\left(\sigma\left(\boldsymbol{x}, \gamma^{h}\right) \mathbf{D} \boldsymbol{u}^{h}\right): \mathbf{D} \boldsymbol{u}^{h}=\int_{0}^{T} \int_{\Omega} \boldsymbol{f} \cdot \boldsymbol{u}^{h}+\int_{0}^{T} \int_{\partial \Omega} \alpha \boldsymbol{u}^{h} \cdot \boldsymbol{u}^{h} .
$$

By the Cauchy-Schwarz and Poincaré inequalities, we deduce the uniform bound

$$
\left\|\boldsymbol{u}^{h}\right\|_{L^{2}\left(0, T ; H^{1}(\Omega)^{3}\right)} \leq \mathcal{C}
$$

here and until the end of the proof, $\mathcal{C}$ is a generic constant that may possibly depend on the $L^{2}$-norms of the initial data and source terms of the system and on the constants appearing in assumptions (E.1)-(E.6), but not on $h$. Then, from the Galerkin formulation and the inf-sup condition (3.1) we derive the uniform $L^{2}$ estimate on $p^{h}$ :

$$
\left\|p^{h}\right\|_{L^{2}\left(\Omega_{T}\right)} \leq \mathcal{C}
$$

Next, we impose the relation (2.15): $v^{h}=v_{e}^{h}-v_{i}^{h}$, and we look at the part (2.14), (2.13), (2.16) of the system. Due to the assumption $V^{h} \subset L^{4}(\Omega)$ we are allowed to take $v_{e}^{h}$ for the test function in (2.14) and $v_{i}^{h}$ for the test function in (2.13) and make the difference; to this, we add (2.16) with the test function $\chi C / A w^{h}$. Notice that due to the time-regularity of $v_{i, e}^{h}$ and $w^{h}$, we do have $\left(\partial_{t} v^{h}\right) v^{h}=\partial_{t}\left(v^{h}\right)^{2} / 2$, $\left(\partial_{t} w^{h}\right) w^{h}=\partial_{t}\left(w^{h}\right)^{2} / 2$. In time, we integrate the equations on $(0, s)$ for every $s<T$, 
which yields the equality

$$
\begin{aligned}
\int_{0}^{s} \int_{\Omega} & \left(\chi\left(j\left(v^{h}\right) v^{h}+\frac{B C}{A}\left(w^{h}\right)^{2}\right)+\left(\mathbf{M}_{e}\left(\boldsymbol{x}, \mathbf{D} \boldsymbol{u}^{h}\right) \nabla v_{e}^{h}\right) \cdot \nabla v_{e}^{h}\right. \\
& \left.+\left(\mathbf{M}_{i}\left(\boldsymbol{x}, \mathbf{D} \boldsymbol{u}^{h}\right) \nabla v_{i}^{h}\right) \cdot \nabla v_{i}^{h}\right)+\int_{\Omega}\left(\frac{c_{m} \chi}{2}\left(v^{h}\right)^{2}(s, \cdot)+\frac{\chi C}{2 A}\left(w^{h}\right)^{2}(s, \cdot)\right) \\
= & \int_{\Omega}\left(\frac{c_{m} \chi}{2}\left(v_{0}^{h}\right)^{2}+\frac{\chi C}{2 A}\left(w_{0}^{h}\right)^{2}\right)+\int_{0}^{s} \int_{\Omega} I_{\mathrm{app}}^{i} v_{i}^{h}-I_{\mathrm{app}}^{e} v_{e}^{h} .
\end{aligned}
$$

Observe that the properties (2.21) of $j$ lead to the lower bound

$$
v j(v) \geq A_{2}^{\prime} v^{4}-A_{1}^{\prime} v^{2},
$$

for some positive constants $A_{1}^{\prime}, A_{2}^{\prime}$. Hence the Gronwall lemma and Poincaré inequality (see, e.g. Ref. 8) applied on (3.4) yields the following uniform in $h$ estimates:

$$
\left\|v_{i, e}^{h}\right\|_{L^{2}\left(0, T ; H^{1}(\Omega)\right)}+\left\|v^{h}\right\|_{L^{4}\left(\Omega_{T}\right)}+\left\|w^{h}\right\|_{L^{2}\left(\Omega_{T}\right)} \leq \mathcal{C}
$$

Finally, from the $L^{2}$ estimate on $v^{h}$ and from the Galerkin approximation of Eq. (2.17) satisfied by $\gamma^{h}$ we deduce a uniform $L^{2}\left(\Omega_{T}\right)$ estimate on $\gamma^{h}$, by taking $\gamma^{h}$ as a test function and by using the Hölder and the Gronwall inequalities.

Further, we introduce the time translates $\left(T^{\tau} v^{h}\right)(t, \cdot):=v^{h}(t+\tau, \cdot)-v^{h}(t, \cdot)$, $\left(T^{\tau} w^{h}\right)(t, \cdot):=w^{h}(t+\tau, \cdot)-w^{h}(t, \cdot)$ and $\left(T^{\tau} \gamma^{h}\right)(t, \cdot):=\gamma^{h}(t+\tau, \cdot)-\gamma^{h}(t, \cdot) ;$ observe that for all $t \in[0, T-\tau]$ these functions take values in $V^{h}$, therefore they can be used as test functions in the Galerkin formulations. The previously proved uniform in $h$ bounds on $w^{h}, \gamma^{h}, \nabla v^{h}$ in $L^{2}\left(\Omega_{T}\right)$ and on $v^{h}$ in $L^{4}\left(\Omega_{T}\right)$ readily yield analogous bounds for the translates $T^{\tau} w^{h}, T^{\tau} \gamma^{h}, \nabla T^{\tau} v^{h}$ and $T^{\tau} v^{h}$, respectively, in the corresponding $L^{2}$ or $L^{4}$ spaces on $(0, T-\tau) \times \Omega$. Following the time compactness technique of Ref. 2, we integrate the Galerkin approximations of (2.14), (2.16), (2.17), respectively, with respect to the time parameter $s \in[t, t+\tau]$ (with $0<\tau<$ $T$ ) and in the resulting equations, we take for the test functions the corresponding translates $T^{\tau} v^{h}, T^{\tau} w^{h}$ and $T^{\tau} \gamma^{h}$, respectively. Keeping in mind the growth bound $|j(v)| \leq C\left(1+|v|^{4}\right)$ for the FitzHugh-Nagumo nonlinearity and the uniform upper bound assumed in (E.3), we apply the Hölder inequality (with $p=4, p^{\prime}=4 / 3$ in the ionic current term and with $p=p^{\prime}=2$ in the other ones). Finally we use the Fubini theorem to interchange the integrals in $t$ and in $s$ and upper bound the resulting terms using the above-mentioned $L^{2}$ and $L^{4}$ bounds and keeping in mind that $\int_{t}^{t+\tau} d s=\tau=\int_{s-\tau}^{s} d t$. In this way, we get the uniform in $h$ bound:

$$
\begin{aligned}
& \int_{0}^{T-\tau} \int_{\Omega}\left(\left|v^{h}(t+\tau, \cdot)-v^{h}(t, \cdot)\right|^{2}+\left|w^{h}(t+\tau, \cdot)-w^{h}(t, \cdot)\right|^{2}\right. \\
& \left.\quad+\left|\gamma^{h}(t+\tau, \cdot)-\gamma^{h}(t, \cdot)\right|^{2}\right) \leq \mathcal{C} \tau .
\end{aligned}
$$


In addition, we get a uniform estimate of space translates of $w^{h}$ and of $\gamma^{h}$ from the uniform $L^{2}$ estimate of $\nabla v^{h}$. Indeed, notice that Eqs. (2.16), (2.17) are linear and $x$ is merely a parameter of the ODEs with respect to $t$. The Galerkin approximations of (2.16), (2.17) take the following form: given $v^{h}: t \rightarrow V^{h}$, $v^{h}(t, x)=\sum_{i=1}^{N^{h}} a_{i}^{h}(t) \phi_{i}(x)$ find $w^{h}=\sum_{i=1}^{N^{h}} b_{i}^{h}(t) \phi_{i}(x), \gamma^{h}=\sum_{i=1}^{N^{h}} c_{i}^{h}(t) \phi_{i}(x)$ such that

$$
\begin{aligned}
& \partial_{t} \int_{\Omega} w^{h} z^{h}-\int_{\Omega} H\left(v^{h}, w^{h}\right) z^{h}=0 \\
& \partial_{t} \int_{\Omega} \gamma^{h} z^{h}-\int_{\Omega} G\left(\gamma^{h}, w^{h}\right) z^{h}=0 \quad \text { for all } z^{h} \in V_{h} .
\end{aligned}
$$

It is readily seen that the linear ODE system

$$
\partial_{t} b^{h}(t)=H\left(a^{h}(t), b^{h}(t)\right), \quad \partial_{t} c^{h}(t)=G\left(c^{h}(t), b^{h}(t)\right) \quad \text { for all } t \in[0, T],
$$

with the appropriate initial data provides the solutions to the above-Galerkin formulation. It follows that the equations

$$
\partial_{t} w^{h}-H\left(v^{h}, w^{h}\right)=0, \quad \partial_{t} \gamma^{h}-G\left(\gamma^{h}, w^{h}\right)=0
$$

are satisfied pointwise in $[0, T] \times \Omega$. Therefore, we inherit ODEs analogous to (3.5), also satisfied pointwise in $[0, T] \times \Omega$, on the space translates $\left(J^{r} w^{h}\right)(\cdot, \boldsymbol{x})=w^{h}(\cdot$, $\boldsymbol{x}+\boldsymbol{r})-w^{h}(\cdot, \boldsymbol{x})$ and $\left(J^{\boldsymbol{r}} \gamma^{h}\right)(\cdot, \boldsymbol{x})=\gamma^{h}(\cdot, \boldsymbol{x}+\boldsymbol{r})-\gamma^{h}(\cdot, \boldsymbol{x})$ (the term $v^{h}$ should be replaced by $\left.\left(J^{\boldsymbol{r}} v^{h}\right)(\cdot, \boldsymbol{x})=v^{h}(\cdot, \boldsymbol{x}+\boldsymbol{r})-v^{h}(\cdot, \boldsymbol{x})\right)$ :

$$
\partial_{t}\left(J^{\boldsymbol{r}} w^{h}\right)-H\left(J^{\boldsymbol{r}} v^{h}, J^{\boldsymbol{r}} w^{h}\right)=0, \quad \partial_{t}\left(J^{\boldsymbol{r}} \gamma^{h}\right)-G\left(J^{\boldsymbol{r}} \gamma^{h}, J^{\boldsymbol{r}} w^{h}\right)=0 .
$$

This system is satisfied pointwise; therefore we can multiply the equations by $J^{r} w^{h}$ and $J^{r} \gamma^{h}$, respectively, and integrate in $x \in \Omega$ and in $t \in[0, T]$. Observe that the $L^{2}\left(0, T ; H^{1}(\Omega)\right)$ estimate of $v^{h}$ we readily get the bound $\int_{0}^{T} \int_{\Omega_{r}}\left|J^{r} v^{h}\right|^{2} \leq \mathcal{C}|\boldsymbol{r}|^{2}$ (here $\boldsymbol{r} \in \mathbb{R}^{3}$ and $\Omega_{\boldsymbol{r}}:=\{\boldsymbol{x} \in \Omega \mid \boldsymbol{x}-\boldsymbol{r} \in \Omega\}$ ). Hence the application of the Young inequality leads to the estimate

$$
\begin{aligned}
& \sup _{0<|\boldsymbol{r}| \leq \delta} \int_{0}^{T} \int_{\Omega_{\boldsymbol{r}}}\left(\left|J^{\boldsymbol{r}} w^{h}\right|^{2}+\left|J^{\boldsymbol{r}} \gamma^{h}\right|^{2}\right) \\
& \quad \leq \mathcal{C}|\boldsymbol{r}|^{2}+T \sup _{0<|\boldsymbol{r}| \leq \delta} \int_{\Omega_{\boldsymbol{r}}}\left(\left|J^{\boldsymbol{r}} w_{0}^{h}\right|^{2}+\left|J^{\boldsymbol{r}} \gamma_{0}^{h}\right|^{2}\right),
\end{aligned}
$$

whose right-hand side vanishes as $|\boldsymbol{r}| \rightarrow 0$, uniformly in $h$.

\subsection{Compactness properties and passage to the limit}

The above estimates imply strong compactness of $\left(v^{h}, w^{h}, \gamma^{h}\right)_{h}$ in $L^{2}((0, T) \times \Omega)$ for all $\tau>0$ and the weak compactness of $\left(v^{h}, v_{i}^{h}, v_{e}^{h}, \boldsymbol{u}^{h}\right)$ in $L^{2}\left(0, T ; H^{1}(\Omega)\right)$. Moreover, the $L^{4}$ estimate of $v^{h}$ and the growth assumption on $j$ contained in (2.21) imply weak compactness of $\left(j\left(v^{h}\right)\right)_{h}$ in the space $L^{4 / 3}\left(\Omega_{T}\right)$ dual to $L^{4}\left(\Omega_{T}\right)$. We proceed by extracting countably many subsequences, assuming that all the above sequences converge to some limits, in the respective strong or weak sense. 
Passage to the limit in the Galerkin formulation for $U^{h}$ is a mere assemblage of classical arguments used separately on the elliptic elasticity equation and then on the bidomain model. Indeed, the electromechanical transmission is ensured via variables $\gamma^{h}, w^{h}$ which behavior is governed by a linear system ODEs, with straightforward passage to the limit. Moreover, due to the convergence (up to extraction of a subsequence) of $\left(\gamma^{h}\right)_{h}$ in the strong $L^{2}$ topology, in a first step we can isolate the passage to the limit in the mechanical part of the system from the behavior of its electrical part. While it is easy to pass to the limit in the linear with respect to $\boldsymbol{u}$ elasticity equations, let us stress that we also recover from this argument the strong $L^{2}$ convergence of the gradients $\left(\mathbf{D} \boldsymbol{u}^{h}\right)_{h}$. Then in a second step, the latter convergence permits to address the passage to the limit in the electrical part of the system, following closely the analysis of Refs. 8 and 5; the only delicate issue here is to identify the weak limit of the nonlinear ionic current term. Observe that the latter issue is addressed in detail in Sec. 5.2 below, in the much more delicate context of semi-implicit in time full discretization of the ionic current term.

Let us give a step-by-step sequence of arguments used for the proof, highlighting the role of the assumptions made in Sec. 2.5.

- The passage to the (weak) limit in the ODEs governing the evolution of $\gamma^{h}, w^{h}$ is straightforward, since they are linear, see assumptions (E.5), (E.6).

- The strong $L^{2}$ convergence on $(0, T-\tau) \times \Omega$ (up to extraction of a subsequence) of $\gamma^{h}$ implies the strong a.e. convergence of the uniformly bounded family of tensors $\sigma\left(\boldsymbol{x}, \gamma^{h}\right)$, due to assumptions (E.1), (E.2).

- With this information, using Lebesgue dominated convergence theorem to deal with the contribution of $\sigma\left(\boldsymbol{x}, \gamma^{h}\right)$ we readily pass to the (weak) limit in the Galerkin formulation of (2.24).

- In addition, because the limit $\boldsymbol{u}$ solves the limit equation (2.12), using the MintyBrowder trick (see, e.g. Refs. 2 and 26$)^{\text {a }}$ we are able to assert that $\mathbf{D} \boldsymbol{u}^{h}$ actually converges to $\mathbf{D} \boldsymbol{u}$ strongly in $L^{2}\left(\Omega_{T}\right)$.

- Due to assumptions (E.1), (E.2), the strong convergence of $\mathbf{D} \boldsymbol{u}^{h}$ implies strong a.e. convergence of $\mathbf{M}_{i, e}\left(\boldsymbol{x}, \mathbf{D} \boldsymbol{u}^{h}\right)$ to the limit $\mathbf{M}_{i, e}(\boldsymbol{x}, \mathbf{D} \boldsymbol{u})$; hence we can use again the dominated convergence argument for the diffusivity tensors involved in the bidomain equations.

- At this point, it is a standard matter to pass to the limit in the variational formulation (2.26), (2.27) of the bidomain equations following the general approach of Ref. 2 (the analogy between the setting of Ref. 2 and the bidomain system was highlighted in Ref. 5, see also Ref. 8). Here, the chain rule in time (cf. Ref. 2) is used in the context of identities $(2.26),(2.27)$ to deal with the contribution of $\partial_{t} v$; the $L^{4 / 3}-L^{4}$ duality is essential to deal with the contribution of the product $j\left(v^{h}\right) v^{h}$, moreover, the FitzHugh-Nagumo assumption (2.21) in (E.6) is exploited, implying that the nonlinearity $j$ is monotone up to a linear term. 


\section{Weak-Strong Comparison and Uniqueness of Regular Solutions}

The strategy for proving uniqueness relies on straightforward estimates on the difference $U-\hat{U}$, in particular, the Lipschitz continuity properties (E.2), (E.4) are instrumental. However, in order to obtain exploitable estimates on $U-\hat{U}$, higher regularity solutions should be considered.

\subsection{Strong-weak uniqueness argument}

Let us first introduce a suitable notion of strong solution, in relation with the uniqueness argument developed in Theorem 4.1 .

Definition 4.1. We will say that $U=\left(\boldsymbol{u}, p, v_{i}, v_{e}, v, w, \gamma\right)$ is a bounded-gradient weak solution ${ }^{\mathrm{b}}$ of problem (2.12)-(2.20) if it is a weak solution in the sense of Definition 2.1 and moreover, one has the following regularity:

$$
\mathbf{D} \boldsymbol{u} \in L^{\infty}\left(\Omega_{T}\right)^{3 \times 3}, \quad \nabla v_{i, e} \in L^{\infty}\left(\Omega_{T}\right)^{3} .
$$

Such regularity of weak solutions is not possible without additional assumptions on the data; clearly, the minimal assumptions include regularity in $\boldsymbol{x}$ of the families of tensors involved in assumptions (E.1), (E.3), regularity of source terms in $t$ and in $\boldsymbol{x}$, and $H^{1}$ regularity of initial data. We refer to Ref. 8 for a result on regularity of bidomain systems; however, the use of its strategy requires at least $L^{1}\left(\Omega_{T}\right)$ estimates of $\partial_{t} \mathbf{D} \boldsymbol{u}$. The latter can be obtained via elliptic regularity and parameter dependence techniques, provided suitable time and space regularity is proved for $\gamma$; in turn, the regularity of $\gamma$ requires that of $v$. We see that simple decoupling strategies cannot be used for justification of $L^{\infty}$ bounds on $\mathbf{D} \boldsymbol{u}, \nabla v_{i, e}$. Yet we think that the following weak-strong uniqueness and continuous dependence result contributes to assess good analytical properties of the model considered herein.

Theorem 4.1. Assume that $U=\left(\boldsymbol{u}, p, v_{i}, v_{e}, v, w, \gamma\right)$ is a bounded-gradient weak solution of problem (2.12)-(2.20) in the sense of the above definition, and $\hat{U}=$ $\left(\hat{\boldsymbol{u}}, \hat{p}, \hat{v}_{i}, \hat{v}_{e}, \hat{v}, \hat{w}, \hat{\gamma}\right)$ is a weak solution in the sense of Definition 2.1 corresponding to the data $\hat{\boldsymbol{f}}, \widehat{I_{\text {app }}^{i}}, \widehat{I_{\text {app }}^{e}}, \hat{v}_{0}, \hat{w}_{0}, \hat{\gamma}_{0}$. Then there exists $\mathcal{K}=\mathcal{K}\left(\|\mathbf{D} \boldsymbol{u}\|_{\infty},\left\|\nabla v_{i, e}\right\|_{\infty}\right)$ (depending also on $T$ and on the different constants involved in assumptions (E.1)-(E.6) and on the initial and source data for both $U$ and $\hat{U})$ such that

$$
\begin{aligned}
\|(v, w, \gamma)-(\hat{v}, \hat{w}, \hat{\gamma})\|_{L^{\infty}\left(0, T ; L^{2}(\Omega)\right)^{3}} & \\
& +\|\boldsymbol{u}-\hat{\boldsymbol{u}}\|_{L^{2}\left(0, T ; H^{1}(\Omega)^{3}\right)}+\|p-\hat{p}\|_{L^{2}\left(\Omega_{T}\right)}+\left\|\left(v_{i}, v_{e}\right)-\left(\hat{v}_{i}, \hat{v}_{e}\right)\right\|_{L^{2}\left(0, T ; H^{1}(\Omega)\right)^{2}} \\
\leq & \mathcal{K}\left(\|\mathbf{D} \boldsymbol{u}\|_{\infty},\left\|\nabla v_{i, e}\right\|_{\infty}\right)\left(\|\boldsymbol{f}-\hat{\boldsymbol{f}}\|_{L^{2}\left(\Omega_{T}\right)^{3}}+\left\|\left(I_{\mathrm{app}}^{i}, I_{\mathrm{app}}^{e}\right)-\left(\widehat{I_{\mathrm{app}}^{i}}, \widehat{I_{\mathrm{app}}^{e}}\right)\right\|_{L^{2}\left(\Omega_{T}\right)^{2}}\right. \\
\quad & \left.+\left\|\left(v_{0}, w_{0}, \gamma_{0}\right)-\left(\hat{v}_{0}, \hat{w}_{0}, \hat{\gamma}_{0}\right)\right\|_{L^{2}(\Omega)^{3}}\right) .
\end{aligned}
$$

In particular, if a bounded-gradient weak solution exists for data $\mathbf{f}, I_{\text {app }}^{i}, I_{\text {app }}^{e}$, $v_{0}, w_{0}, \gamma_{0}$, then it is a unique weak solution to the problem.

\footnotetext{
${ }^{\mathrm{b}} \mathrm{A}$ related concept of bounded-gradient solutions can be found in e.g. Ref. 25 .
} 
Proof. Consider Eq. (2.24) written for $\boldsymbol{u}$ and $\hat{\boldsymbol{u}}$; using $\boldsymbol{u}-\hat{\boldsymbol{u}}$ as test function, we obtain

$$
\begin{aligned}
& (\sigma(\boldsymbol{x}, \gamma) \mathbf{D} \boldsymbol{u}-\sigma(\boldsymbol{x}, \hat{\gamma}) \mathbf{D} \hat{\boldsymbol{u}}):(\mathbf{D} \boldsymbol{u}-\mathbf{D} \hat{\boldsymbol{u}}) \\
& \quad=(\sigma(\boldsymbol{x}, \hat{\gamma})(\mathbf{D} \boldsymbol{u}-\mathbf{D} \hat{\boldsymbol{u}})):(\mathbf{D} \boldsymbol{u}-\mathbf{D} \hat{\boldsymbol{u}})+((\sigma(\boldsymbol{x}, \gamma)-\sigma(\boldsymbol{x}, \hat{\gamma})) \mathbf{D} \boldsymbol{u}):(\mathbf{D} \boldsymbol{u}-\mathbf{D} \hat{\boldsymbol{u}})
\end{aligned}
$$

Then, thanks to Young inequality, the coercivity assumption (E.1), and the Lipschitz continuity (E.2), we obtain for all $t \in(0, T)$,

$$
\begin{aligned}
\int_{\partial \Omega} & \frac{\alpha}{2}|\boldsymbol{u}-\hat{\boldsymbol{u}}|^{2}(t)+\int_{\Omega} \frac{1}{2 c}|\mathbf{D} \boldsymbol{u}-\mathbf{D} \hat{\boldsymbol{u}}|^{2}(t) \\
& \leq \mathcal{C}\left(\int_{\Omega}|\boldsymbol{f}-\hat{\boldsymbol{f}}|^{2}(t)+\|\mathbf{D} \boldsymbol{u}\|_{L^{\infty}\left(\Omega_{T}\right)} \int_{\Omega}|\gamma-\hat{\gamma}|^{2}(t)\right) .
\end{aligned}
$$

Here and in the sequel, $\mathcal{C}$ is a generic constant depending on the data of both systems (for $U$ and $\hat{U}$ ) and on the constants in (E.1)-(E.6). Similarly, we use the ODEs (2.17) for both $\gamma$ and $\hat{\gamma}$ with test function $\gamma-\hat{\gamma}$ and deduce

$$
\int_{\Omega}|\gamma-\hat{\gamma}|^{2}(t) \leq \mathcal{C}\left(\int_{\Omega}\left|\gamma_{0}-\hat{\gamma}_{0}\right|^{2}+\int_{0}^{t} \int_{\Omega}|w-\hat{w}|^{2}(s) d s\right) .
$$

Finally, we combine Eqs. (2.13)-(2.16) written for $U$ and $\hat{U}$ in the same way as for the proof of (3.4); we use Lemma 2.3 in Ref. 5 in order to justify the possibility to take test functions $v_{i, e}-\hat{v}_{i, e}$ in (2.13), (2.14), respectively. Rearranging the terms as in (4.1), using in addition the fact that

$$
(v \mathcal{B}(v)-\hat{v} \mathcal{B}(\hat{v}))(v-\hat{v}) \geq 0,
$$

where $\mathcal{B}$ is defined as in $(2.22)$, we find for a.e. $t \in(0, T)$ the following bound:

$$
\begin{aligned}
\int_{\Omega}\left(\frac{c_{m} \chi}{2}|v-\hat{v}|^{2}(t)+\frac{B C}{A}|w-\hat{w}|^{2}(t)\right) \\
\quad+\frac{1}{2 c} \int_{0}^{t} \int_{\Omega}\left(\left|\nabla v_{i}-\nabla \hat{v}_{i}\right|^{2}(s)+\left|\nabla v_{e}-\nabla \hat{v}_{e}\right|^{2}(s) d s\right) \\
\leq \mathcal{C}\left(\int_{\Omega}\left(\| v_{0}-\left.\hat{v}_{0}\right|^{2}+\left|w_{0}-\hat{w}_{0}\right|^{2}\right)+\int_{0}^{t} \int_{\Omega}\left|\left(I_{\mathrm{app}}^{i}, I_{\mathrm{app}}^{e}\right)-\left(\widehat{I_{\mathrm{app}}^{i}}, \widehat{I_{\mathrm{app}}^{e}}\right)\right|^{2}(s) d s\right. \\
\quad+\left\|\left(\nabla v_{i}, \nabla v_{e}\right)\right\|_{L^{\infty}\left(\Omega_{T}\right)^{2 \times 3}} \int_{0}^{t} \int_{\Omega}|\mathbf{D} \boldsymbol{u}-\mathbf{D} \hat{\boldsymbol{u}}|^{2}(s) d s \\
\left.\quad+\int_{0}^{t} \int_{\Omega}\left(|v-\hat{v}|^{2}(s)+|w-\hat{w}|^{2}(s)\right) d s\right) .
\end{aligned}
$$

Then it is straightforward to combine (4.2)-(4.4) and obtain a Gronwall-type inequality that bounds $U-\hat{U}$ in the way stated in the theorem. Indeed, we can set:

$$
\begin{aligned}
E(t) & :=\|\mathbf{D} \boldsymbol{u}-\mathbf{D} \hat{\boldsymbol{u}}\|_{L^{2}(\Omega)^{3 \times 3}}^{2}(t), \quad \Gamma(t):=\|\gamma-\hat{\gamma}\|_{L^{2}(\Omega)}^{2}(t), \\
W(t) & :=\frac{c_{m} \chi}{2}\|v-\hat{v}\|_{L^{2}(\Omega)}^{2}(t)+\frac{B C}{A}\|w-\hat{w}\|_{L^{2}(\Omega)}^{2}(t),
\end{aligned}
$$




$$
\begin{aligned}
D(t) & :=\|\boldsymbol{f}-\hat{\boldsymbol{f}}\|_{L^{2}(\Omega)^{3}}^{2}(t)+\left\|\left(I_{\mathrm{app}}^{i}, I_{\mathrm{app}}^{e}\right)-\left(\widehat{I_{\mathrm{app}}^{i}}, \widehat{I_{\mathrm{app}}^{e}}\right)\right\|_{L^{2}(\Omega)^{2}}^{2}(t), \\
D_{0} & :=\left\|\left(v_{0}, w_{0}, \gamma_{0}\right)-\left(\hat{v}_{0}, \hat{w}_{0}, \hat{\gamma}_{0}\right)\right\|_{L^{2}(\Omega)^{3}}^{2} .
\end{aligned}
$$

Dropping non-negative terms, from (4.2), (4.3) and (4.4) we infer:

$$
\begin{aligned}
E(t) & \leq \mathcal{K}(D(t)+\Gamma(t)), \\
\Gamma(t) & \leq \mathcal{K}\left(D_{0}+\int_{0}^{t} W(s) d s\right), \\
W(t) & \leq \mathcal{K}\left(D_{0}+\int_{0}^{t} D(s) d s+\int_{0}^{t} E(s) d s+\int_{0}^{t} W(s) d s\right),
\end{aligned}
$$

with a generic constant $\mathcal{K}$ depending on the different constants, data and solutions as indicated in the statement of the theorem. Hence, substituting the second inequality in the first one, then substituting the resulting inequality into the third one and applying the Fubini theorem in the double integral $\int_{0}^{t} \int_{0}^{s} W(\tau) d \tau d s$ that appears from this calculation, we find

$$
W(t) \leq \mathcal{K}\left(D_{0}+\int_{0}^{t} D(s) d s+\int_{0}^{t}(1+(t-s)) W(s) d s\right), \quad W(0) \leq \mathcal{C} D_{0} .
$$

Then we can apply the Gronwall lemma (see, e.g. Ref. 37); this permits to bound $\|(v, w)-(\hat{v}, \hat{w})\|_{L^{2}(\Omega)^{2}}(t)$ as claimed in Theorem 4.1. Then, with the help of (4.3) we also bound $\|\gamma-\hat{\gamma}\|_{L^{2}(\Omega)}(t)$. Next, with (4.2) we bound $\|\mathbf{D} \boldsymbol{u}-\mathbf{D} \hat{\boldsymbol{u}}\|_{L^{2}(\Omega)^{3 \times 3}}(t)$ and $\|\boldsymbol{u}-\hat{\boldsymbol{u}}\|_{L^{2}(\partial \Omega)^{3}}(t)$, which implies the $H^{1}(\Omega)^{3}$ bound on $(\boldsymbol{u}-\hat{\boldsymbol{u}})(t)$, thanks to Poincaré inequality. Now, the bound on $\|p-\hat{p}\|_{L^{2}(\Omega)}(t)$ follows from $(2.12)$ written for $\boldsymbol{u}(t)$ and $\hat{\boldsymbol{u}}(t)$, using a test function $\boldsymbol{v} \in H^{1}(\Omega)$ such that $\nabla \cdot \boldsymbol{v}=p(t)-\hat{p}(t)$. Such a function can be constructed for each $t$ by taking $\boldsymbol{v}=\nabla \theta$ using the auxiliary Dirichlet problem $-\Delta \theta=(p-\hat{p})$ in $\tilde{\Omega}, \theta=0$ on $\partial \tilde{\Omega}$, where $\tilde{\Omega}$ is a regular domain containing $\Omega$; we have $\theta \in H^{2}(\tilde{\Omega})$ by the classical elliptic regularity results. Finally, with (4.4) we also achieve the desired bounds on $\left\|\nabla v_{i, e}-\nabla \hat{v}_{i, e}\right\|_{L^{2}(\Omega)}(t)$.

\section{Numerical Approximation}

Here, we present the finite element method for approximation of the problem studied in the previous section, the associated numerical results and a critical analysis of validity of the approximations made in Sec. 2.4. Other discretization techniques can certainly be employed, following e.g. Refs. 16, 17, 19 and 44 .

\subsection{A finite element method}

Let $\mathcal{T}_{h}$ be a regular partition of $\Omega$ into tetrahedra $K$ with boundary $\partial K$ and diameter $h_{K}$. We define the mesh parameter $h=\max _{K \in \mathcal{T}_{h}}\left\{h_{K}\right\}$ and the associated finite element spaces $V^{h}$ (respectively, $\mathcal{V}^{h}$ ), for the approximation of pressure, electrical potentials and ionic variables (respectively, of displacements). In order to satisfy the discrete Ladyzhenskaya-Babuška-Brezzi stability condition (3.1), piecewise 
quadratic finite elements are used to approximate the displacements field, while for pressure, electrical potentials and ionic variables, we use piecewise linear elements. That is, the involved spaces are defined as

$$
\begin{aligned}
& V^{h}=\left\{s \in C^{0}(\bar{\Omega}):\left.v\right|_{K} \in \mathbb{P}_{1}(K) \text { for all } K \in \mathcal{T}_{h}\right\}, \\
& \mathcal{V}^{h}=\left\{\boldsymbol{v} \in C^{0}(\bar{\Omega}):\left.\boldsymbol{v}\right|_{K} \in \mathbb{P}_{2}(K)^{3} \text { for all } K \in \mathcal{T}_{h}\right\}
\end{aligned}
$$

In order to lighten the notation, in this section we put $\chi=c_{m}=1$ (the general case is completely analogous). The semi-discrete Galerkin finite element formulation used in Sec. 3.1 then reads: for $t>0$, find $\boldsymbol{u}^{h} \in \mathcal{V}^{h}, v_{i}^{h}(t), v_{e}^{h}(t), v^{h}(t)$, $w^{h}(t), \gamma^{h}(t), p^{h}(t) \in V^{h}$ such that (with the standard finite element notation for $L^{2}$ scalar products) one has:

$$
\begin{aligned}
\left(\boldsymbol{\sigma}\left(\boldsymbol{x}, \gamma^{h}(t)\right) \mathbf{D} \boldsymbol{u}^{h}(t), \mathbf{D} \boldsymbol{\psi}^{h}\right)_{\Omega}-\left(p^{h}(t), \nabla \cdot \boldsymbol{\psi}^{h}\right)_{\Omega} & =\left(\boldsymbol{f}^{h}, \boldsymbol{\psi}^{h}\right)_{\Omega}+\left(\alpha \boldsymbol{u}^{h}(t), \boldsymbol{\psi}^{h}\right)_{\partial \Omega}, \\
\left(\boldsymbol{u}^{h}(t), \nabla \phi^{h}\right)_{\Omega} & =0 \\
\frac{d}{d t}\left(v^{h}(t), \phi^{h}\right)_{\Omega}+\left(\mathbf{M}_{i}\left(\boldsymbol{x}, \mathbf{D} \boldsymbol{u}^{h}(t)\right) \nabla v_{i}^{h}(t), \nabla \phi^{h}\right)_{\Omega} & =\left(I_{\mathrm{app}}^{i}-I_{\mathrm{ion}}\left(v^{h}(t), w^{h}(t)\right), \phi^{h}\right)_{\Omega}, \\
\frac{d}{d t}\left(v^{h}(t), \phi^{h}\right)_{\Omega}-\left(\mathbf{M}_{e}\left(\boldsymbol{x}, \mathbf{D} \boldsymbol{u}^{h}(t)\right) \nabla v_{e}^{h}(t), \nabla \phi^{h}\right)_{\Omega} & =\left(I_{\mathrm{app}}^{e}-I_{\mathrm{ion}}\left(v^{h}(t), w^{h}(t)\right), \phi^{h}\right)_{\Omega}, \\
\frac{d}{d t}\left(w^{h}(t), \phi^{h}\right)_{\Omega} & =\left(H\left(v^{h}(t), w^{h}(t)\right), \phi^{h}\right)_{\Omega}, \\
\frac{d}{d t}\left(\gamma^{h}(t), \phi^{h}\right)_{\Omega} & =\left(G\left(\gamma^{h}(t), w^{h}(t)\right), \phi^{h}\right)_{\Omega},
\end{aligned}
$$

for all $\boldsymbol{\psi}^{h} \in \mathcal{V}^{h}$ and all $\phi^{h} \in V^{h}$; one also sets $v^{h}(0)=\mathbb{P}_{V^{h}}\left(v_{0}\right)$ (analogous initialization is used for $w^{h}$ and $\left.\gamma^{h}\right)$. A classical backward Euler integration method is employed for the time discretization of (5.1) with time step $\delta t=T / N$, moreover, we linearize and decouple the resulting time-implicit scheme by employing $\gamma^{h, n-1}$ (respectively, $\mathbf{D} \boldsymbol{u}^{h, n-1}$ ) in the equation on $\boldsymbol{u}^{h, n}$ (respectively, in the equations on $\left.v_{i, e}^{h, n}\right)$ and by linearizing $I_{\text {ion }}$ with the help of the new function

$$
I_{\text {ion }}^{\operatorname{lin}}:(v, \tilde{v}, w) \mapsto v \mathcal{B}(\tilde{v})-A_{1} v+C w \quad \text { where } \quad \mathcal{B}: z \mapsto \frac{j(z)}{z}+A_{1} \geq 0 .
$$

This results in the following fully discrete method: find $v_{i}^{h}(t), v_{e}^{h}(t), v^{h}(t), w^{h}(t)$, $\gamma^{h}(t), p^{h}(t) \in V^{h}$ and $\boldsymbol{u}^{h} \in \mathcal{V}^{h}$ such that:

$$
\begin{gathered}
\boldsymbol{u}^{h}(t, \boldsymbol{x})=\sum_{n=1}^{N} \boldsymbol{u}^{h, n}(\boldsymbol{x}) \mathbb{1}_{((n-1) \delta t, n \delta t]}(t), \\
\left(v_{i}^{h}, v_{e}^{h}, v^{h}, w^{h}, \gamma^{h}, p^{h}\right)(t, \boldsymbol{x})=\sum_{n=1}^{N}\left(v_{i}^{h, n}, v_{e}^{h, n}, v^{h, n}, w^{h, n}, \gamma^{h, n}, p^{h, n}\right)(\boldsymbol{x}) \mathbb{1}_{((n-1) \delta t, n \delta t]}(t),
\end{gathered}
$$


satisfy the algebraic system of equations:

$$
\begin{aligned}
& \left(\boldsymbol{\sigma}\left(\boldsymbol{x}, \gamma^{h, n-1}\right) \mathbf{D} \boldsymbol{u}^{h, n}, \mathbf{D} \boldsymbol{\psi}^{h}\right)_{\Omega}+\left(p^{h, n}, \nabla \cdot \boldsymbol{\psi}^{h}\right)_{\Omega}=\left(\boldsymbol{f}^{h, n}, \boldsymbol{\psi}^{h}\right)_{\Omega}+\left(\alpha \boldsymbol{u}^{h, n}, \boldsymbol{\psi}^{h}\right)_{\partial \Omega}, \\
& \left(\boldsymbol{u}^{h, n}, \nabla \phi^{h}\right)_{\Omega}=0, \\
& \left(\frac{v^{h, n}-v^{h, n-1}}{\delta t}, \phi^{h}\right)_{\Omega}+\left(\mathbf{M}_{i}\left(\boldsymbol{x}, \mathbf{D} \boldsymbol{u}^{h, n-1}\right) \nabla v_{i}^{h, n}, \nabla \phi^{h}\right)_{\Omega} \\
& =\left(I_{\text {app }}^{i}-I_{\text {ion }}^{\operatorname{lin}}\left(v^{h, n}, v^{h, n-1}, w^{h, n}\right), \phi^{h}\right)_{\Omega}, \\
& \left(\frac{v^{h, n}-v^{h, n-1}}{\delta t}, \phi^{h}\right)_{\Omega}-\left(\mathbf{M}_{e}\left(\boldsymbol{x}, \mathbf{D} \boldsymbol{u}^{h, n-1}\right) \nabla v_{e}^{h, n}, \nabla \phi^{h}\right)_{\Omega} \\
& =\left(I_{\text {app }}^{e}-I_{\text {ion }}^{\operatorname{lin}}\left(v^{h, n}, v^{h, n-1}, w^{h, n}\right), \phi^{h}\right)_{\Omega}, \\
& \left(\frac{w^{h, n}-w^{h, n-1}}{\delta t}, \phi^{h}\right)_{\Omega}=\left(H\left(v^{h, n}, w^{h, n}\right)_{,} \phi^{h}\right)_{\Omega}, \\
& \left(\frac{\gamma^{h, n}-\gamma^{h, n-1}}{\delta t}, \phi^{h}\right)_{\Omega}=\left(G\left(\gamma^{h, n}, w^{h, n}\right), \phi^{h}\right)_{\Omega},
\end{aligned}
$$

for all $\boldsymbol{\psi}^{h} \in \mathcal{V}^{h}, \phi^{h} \in V^{h}$ and for all $n \in\{1, \ldots, N\}$; the initial condition takes the form

$$
\left(v^{h, 0}, w^{h, 0}, \gamma^{h, 0}\right)=\left(\mathbb{P}_{V^{h}}\left(v_{0}\right), \mathbb{P}_{V^{h}}\left(w_{0}\right), \mathbb{P}_{V^{h}}\left(\gamma_{0}\right)\right)
$$

Here $I_{\text {app }}^{i, h}(\cdot), I_{\text {app }}^{e, h}(\cdot)$ are time averages over $[(n-1) \delta t, n \delta t]$ of $I_{\text {app }}^{i}, I_{\text {app }}^{e}$, respectively.

\subsection{A glimpse into the convergence proof}

Consider solutions to the fully discrete system, and let us indicate some milestones of its convergence analysis. The proof of stability estimates closely follows the one presented in Sec. 3.2 above for semi-discrete Galerkin approximations. Namely, we use the same kind of test functions in the same combination of equations; instead of the chain rule for time derivatives used in the continuous setting, the convexity inequality

$$
\left(a^{n}-a^{n-1}\right) a^{n} \geq \frac{\left(a^{n}\right)^{2}}{2}-\frac{\left(a^{n-1}\right)^{2}}{2}
$$

is used to deal with the finite differences in time. In this way, we obtain uniform in $h$ and $\delta t$ estimates on the discrete solutions:

$\left\|\boldsymbol{u}^{h}\right\|_{L^{2}\left(0, T ; H^{1}(\Omega)^{3}\right)}+\left\|p^{h}\right\|_{L^{2}\left(\Omega_{T}\right)}+\left\|v_{i, e}^{h}\right\|_{L^{2}\left(0, T ; H^{1}(\Omega)\right)}+\left\|\left(v^{h}, w^{h}, \gamma^{h}\right)\right\|_{L^{2}\left(\Omega_{T}\right)} \leq \mathcal{C}$,

and also the following uniform in $h$ estimate related to linearization of the nonlinearity $I_{\text {ion }}$,

$$
\sum_{n=1}^{N} \delta t \int_{\Omega}\left|v^{h, n}\right|^{2} \mathcal{B}\left(v^{h, n-1}\right) \leq \mathcal{C} .
$$


The latter estimate replaces, in a weaker sense, the $L^{4}\left(\Omega_{T}\right)$ estimate of $v^{h}$ obtained in the semi-discrete Galerkin context. The details of these estimates, including the latter one, are also very close to those given in Ref. 5 where a linearized implicit finite volume scheme of bidomain equation is analyzed. Estimate (5.2) implies in particular the invertibility of the matrix of the discrete system that is solved on each time step.

Now, similarly to Sec. 3.2 we get space and time translation estimates on $\left(w^{h}\right)_{h}$ and on $\left(\gamma^{h}\right)_{h}$. For the time estimates, we introduce, e.g. $\bar{w}^{h}$ the piecewise affine in $t$ function in $W^{1, \infty}\left([0, T] ; V^{h}\right)$ interpolating the states $\left(w^{h, n}\right)_{n=0 \cdots N} \subset V^{h}$ at the points $(n \delta t)_{n=0 \cdots N}$. Then we have $\bar{w}_{t}^{h}=H\left(v^{h}, w^{h}\right)$, and due to the linearity of $H$, with the notation introduced in Sec. 3.2 we also have

$$
\left(T^{\tau} \bar{w}^{h}\right)_{t}=H\left(T^{\tau} v^{h}, T^{\tau} w^{h}\right) .
$$

We can use test function $\left(T^{\tau} w^{h}\right)(t, \cdot)$ in this equation to obtain time translation estimates on $\left(\bar{w}^{h}\right)_{h}$ using Fubini theorem and the bounds (5.2). Further, the estimates of space translates $\left(J^{\mathbf{r}} w^{h}\right)_{h}$ are obtained in the same way as (3.7), hence analogous estimate on $\left(J^{\mathbf{r}} \bar{w}^{h}\right)_{h}$ follows because for $t \in((n-1) \delta t, n \delta t], \bar{w}^{h}(t, \cdot)$ is a convex combination of $w^{h, n-1}(\cdot)$ and $w^{h, n}(\cdot)$. By the Fréchet-Kolmogorov theorem we deduce strong $L^{2}\left(\Omega_{T}\right)$ compactness of $\left(\bar{w}^{h}\right)_{h}$. Further, it is easily seen from the definition of $\bar{w}^{h}$, from the equation

$$
\frac{w^{h, n}-w^{h, n-1}}{\delta t}=H\left(v^{h, n}, w^{h, n}\right),
$$

and estimates (5.2) that

$$
\left\|\bar{w}^{h}-w^{h}\right\|_{L^{2}\left(\Omega_{T}\right)}^{2} \leq \sum_{n=1}^{N} \delta t\left\|w^{h, n-1}-w^{h, n}\right\|_{L^{2}(\Omega)}^{2} \leq \mathcal{C} \delta t \rightarrow 0 \quad \text { as } \delta t \rightarrow 0 .
$$

Finally, we conclude that $\left(w^{h}\right)_{h}$ is strongly compact in $L^{2}\left(\Omega_{T}\right)$. The same argument applies to $\left(\gamma^{h}\right)_{h}$. Unfortunately, the lack of $L^{4}\left(\Omega_{T}\right)$ estimate on $\left(v^{h}\right)_{h}$ precludes us from getting the analogous time translation estimate and compactness property on $\left(v^{h}\right)_{h}$, but we circumvent this difficulty using the Young measures' representation of weakly convergent sequences, in the spirit of Ref. 21.

Indeed, extracting convergent subsequences corresponding to the above arguments, firstly we pass to the limit in the linear ODEs and in the elliptic equation on $\boldsymbol{u}^{h}$; as in Sec. 3.3, we get strong convergence of $\mathbf{D} \boldsymbol{u}^{h}$ to $\mathbf{D} \boldsymbol{u}$ from the Minty-Browder argument. Now, we pass to the limit in the equations on $v_{i, e}^{h}$; the delicate point is the passage to the limit in the nonlinearity $I_{\mathrm{ion}}^{\mathrm{lin}}\left(v^{h}, \tilde{v}^{h}, w^{h}\right)$, where $\tilde{v}^{h}(t, \boldsymbol{x})=v^{h}(t-\delta t, \boldsymbol{x})$ with the convention that $v^{h}(t, \boldsymbol{x})=v^{h, 0}(\boldsymbol{x})$ for $t \leq 0$. More precisely, we have to pass to the limit in the term $v^{h} \mathcal{B}\left(\tilde{v}^{h}\right)$. Here we exploit estimate (5.3) to get an equi-integrability estimate on this term. As in Ref. 5, using the Sobolev embedding of $L^{2}\left(0, T ; H^{1}(\Omega)\right)$ into $L^{2}\left(0, T ; L^{6}(\Omega)\right)$ and the space interpolation with $L^{\infty}\left(0, T ; L^{2}(\Omega)\right)$, we find a uniform $L^{10 / 3}\left(\Omega_{T}\right)$ bound on $v_{h}$. Such a bound yields equi-integrability of $\left|\tilde{v}^{h}\right|^{2}$ on $\Omega_{T}$, thus from the weighted Young 
inequality

$$
|v \mathcal{B}(\tilde{v})| \leq \delta|v|^{2} \mathcal{B}(\tilde{v})+\frac{1}{\delta} \mathcal{B}(\tilde{v}),
$$

using the growth assumption on $j(\cdot)$ that implies that $\mathcal{B}(\tilde{v}) \leq \mathcal{C}\left(1+|\tilde{v}|^{2}\right)$, we derive a uniform $L^{1}\left(\Omega_{T}\right)$ bound and, moreover, the equi-integrability on $\Omega_{T}$ for the family $\left(v^{h} \mathcal{B}\left(\tilde{v}^{h}\right)\right)_{h}$. Therefore up to extraction of a further subsequence, $v^{h} \mathcal{B}\left(\tilde{v}^{h}\right)$ converges weakly in $L^{1}\left(\Omega_{T}\right)$ to a limit that we denote by $\Psi$. Further, multiplying the discrete equation for $v^{h, n}$ by $\frac{1}{2}\left(v^{h, n}+v^{h, n-1}\right)$ and summing in $n$ from the previously obtained estimates one deduces the uniform in $h$ estimate

$$
\left|\sum_{n=1}^{N} \delta t \int_{\Omega} v^{h, n} v^{h, n-1} \mathcal{B}\left(v^{h, n-1}\right)\right| \leq \mathcal{C} .
$$

Finally, multiplying the same equation for $v^{h, n}$ by $\left(v^{h, n}-v^{h, n-1}\right)$ and summing in $n$, using in particular (5.4), we get

$$
\sum_{n=1}^{N} \delta t \int_{\Omega}\left|v^{h, n}-v^{h, n-1}\right|^{2} \rightarrow 0 \quad \text { as } \delta t \rightarrow 0 .
$$

Therefore, $\tilde{v}^{h}-v^{h}$ converges to zero strongly in $L^{2}\left(\Omega_{T}\right)$, in particular, the weak $L^{2}\left(\Omega_{T}\right)$ limit $v$ of $v^{h}$ is also the weak $L^{2}\left(\Omega_{T}\right)$ limit of $\tilde{v}^{h}$. Now, in order to prove strong convergence of $\left(v^{h}\right)_{h}$ and to pass to the limit in the nonlinear ionic current term $v^{h} \mathcal{B}\left(\tilde{v}^{h}\right)$ (we have to prove in particular that $\Psi=v \mathcal{B}(v)$ ), we introduce the Young measure $\left(\nu_{(t, \boldsymbol{x})}\right)_{(t, \boldsymbol{x}) \in \Omega_{T}}$ which is the limit of the selected subsequence of $\left(v^{h}\right)_{h}$ (not relabeled):

$v(t, \boldsymbol{x})=\int_{\mathbb{R}} \lambda d \nu_{(t, \boldsymbol{x})}(\lambda) \quad$ with $\quad \int_{\mathbb{R}} d \nu_{(t, \boldsymbol{x})}(\lambda)=1 \quad$ for a.e. $(t, \boldsymbol{x}) \in \Omega_{T}$, moreover,

for all $F \in C^{0}(\mathbb{R} ; \mathbb{R})$ such that $\left(F\left(v^{h}\right)\right)_{h}$ is weakly convergent in $L^{1}\left(\Omega_{T}\right)$,

$$
F\left(v^{h}\right) \rightarrow \int_{\mathbb{R}} F(\lambda) d \nu_{(\cdot, \cdot)}(\lambda) \quad \text { in } L^{1}\left(\Omega_{T}\right)
$$

Then the convergence proof relies on the following observations.

Lemma 5.1. The Young measure $\left(\nu_{(t, \boldsymbol{x})}\right)_{(t, \boldsymbol{x}) \in \Omega_{T}}$ has the following properties:

(i) $v(t, \boldsymbol{x})=\int_{\mathbb{R}} \lambda d \nu_{(t, \boldsymbol{x})}(\lambda)$ and $\Psi(t, \boldsymbol{x})=\int_{\mathbb{R}} \lambda \mathcal{B}(\lambda) d \nu_{(t, \boldsymbol{x})}(\lambda)$;

(ii) $\iint_{\Omega_{T}} \int_{\mathbb{R}} \lambda^{2} \mathcal{B}(\lambda) d \nu_{(t, \boldsymbol{x})}(\lambda) d \boldsymbol{x} d t<\infty$ and $v \in L^{4}\left(\Omega_{T}\right), \Psi \in L^{4 / 3}\left(\Omega_{T}\right)$;

(iii) for a.e. $(t, \boldsymbol{x}) \in \Omega_{T}, \int_{\mathbb{R}} \int_{\mathbb{R}}(\lambda-\mu)(\lambda \mathcal{B}(\lambda)-\mu \mathcal{B}(\mu)) d \nu_{(t, \boldsymbol{x})}(\lambda) d \nu_{(t, \boldsymbol{x})}(\mu) \leq 0$;

(iv) for a.e. $(t, \boldsymbol{x}) \in \Omega_{T}, \nu_{(t, \boldsymbol{x})}(\lambda)=\delta(\lambda-v(t, \boldsymbol{x}))$; in particular, $v^{h} \rightarrow v$ and $\tilde{v}^{h} \rightarrow v$ a.e. on $\Omega_{T}$, and we have $\Psi=v \mathcal{B}(v)$ a.e. on $\Omega_{T}$.

Proof. For (i) and (ii) we use the estimates established above, while for (iii), we use the equations satisfied by $v^{h}$ and by $v$ in a way similar to the Minty trick. The last point follows by classical properties of Young measures. 
(i) The first claim of (i) is a part of the definition of the Young measure. To prove the second point, we first observe that $\Delta_{1}^{h}:=v^{h} \mathcal{B}\left(\tilde{v}^{h}\right)-v^{h} \mathcal{B}\left(v^{h}\right)$ tends to zero in $L^{1}\left(\Omega_{T}\right)$ as $h \rightarrow 0$. Indeed, recall that $v^{h}, \tilde{v}^{h}$ are bounded in $L^{10 / 3}\left(\Omega_{T}\right)$ due to interpolation and embedding results; therefore $\Delta_{1}^{h}$ is bounded in $L^{10 / 9}\left(\Omega_{T}\right)$, and thus it is equi-integrable. Moreover, extracting a further subsequence we can assume that $v^{h}-\tilde{v}^{h} \rightarrow 0$ a.e. on $\Omega_{T}$, so that $\Delta_{1}^{h} \rightarrow 0$ a.e. on $\Omega_{T}$. Then $\Delta_{1}^{h}$ vanishes in $L^{1}\left(\Omega_{T}\right)$ due to the Vitali theorem. Now, we have $v^{h} \mathcal{B}\left(\tilde{v}^{h}\right) \rightarrow \Psi$ in $L^{1}\left(\Omega_{T}\right)$. Consequently, we have as well $v^{h} \mathcal{B}\left(v^{h}\right) \rightarrow \Psi$ in $L^{1}\left(\Omega_{T}\right)$ as $h \rightarrow 0$, and we can apply the representation formula of $(5.5)$ to $\Psi$.

(ii) Consider a family of bounded continuous functions $\left(\mathcal{B}_{n}\right)_{n}$ on $\mathbb{R}$ that increase towards the limit $\mathcal{B}$ pointwise on $\mathbb{R}$, as $n \rightarrow \infty$. Then $F_{n}: \lambda \mapsto \lambda^{2} \mathcal{B}_{n}(\lambda)$ can be used in (5.5) because we know that $\left(v^{h}\right)_{h}$ is an equi-integrable sequence on $\Omega_{T}$ and $b_{n}$ is bounded for every fixed $n$. In addition, as in the point (i) we readily see that $\Delta_{2}^{h}:=\left(v^{h}\right)^{2} \mathcal{B}_{n}\left(v^{h}\right)-\left(v^{h}\right)^{2} \mathcal{B}_{n}\left(\tilde{v}^{h}\right)$ vanishes in $L^{1}\left(\Omega_{T}\right)$ as $h \rightarrow 0$, up to a subsequence. Therefore we find, with the limits taken in the weak $L^{1}\left(\Omega_{T}\right)$ sense, the chain of equalities

$$
\int_{\mathbb{R}} F_{n}(\lambda) d \nu_{(\cdot, \cdot)}(\lambda)=\lim _{h \rightarrow 0} F_{n}\left(v^{h}\right)=\lim _{h \rightarrow 0}\left(v^{h}\right)^{2} \mathcal{B}_{n}\left(\tilde{v}^{h}\right) .
$$

In particular,

$$
\begin{aligned}
\iint_{\Omega_{T}} \int_{\mathbb{R}} F_{n}(\lambda) d \nu_{(t, \boldsymbol{x})}(\lambda) d t d \boldsymbol{x} & \leq \lim _{h \rightarrow 0} \iint_{\Omega_{T}}\left(v^{h}\right)^{2} \mathcal{B}_{n}\left(\tilde{v}^{h}\right) \\
& \leq \iint_{\Omega_{T}}\left(v^{h}\right)^{2} \mathcal{B}\left(\tilde{v}^{h}\right) \\
& \leq \mathcal{C}
\end{aligned}
$$

due to estimate (5.3). Notice that $\mathcal{C}$ does not depend on $n$, therefore using the monotonicity of $\left(\mathcal{B}_{n}\right)_{n}$ we deduce the first claim of (ii) from the monotone convergence theorem. Then, the lower growth bound on $\mathcal{B}$ yields $\int_{\mathbb{R}} \lambda^{4} d \nu_{(\cdot, \cdot)}(\lambda) \in L^{1}\left(\Omega_{T}\right)$, whence by the Jensen inequality for the convex function $\lambda \mapsto \lambda^{4}$ and the probability measures $\nu_{(t, \boldsymbol{x})}$ we deduce that $v=\int_{\mathbb{R}} \lambda d \nu_{(\cdot, \cdot)}(\lambda) \in L^{4}\left(\Omega_{T}\right)$. Using the upper growth bound on $\mathcal{B}$ we find that $\Psi \in L^{3 / 4}\left(\Omega_{T}\right)$.

(iii) Firstly, we prove the inequality

$$
\limsup _{h \rightarrow 0} \iint_{\Omega_{T}}(T-t)\left(v^{h}\right)^{2} \mathcal{B}\left(\tilde{v}^{h}\right) \leq \iint_{\Omega_{T}}(T-t) v \Psi,
$$

for sufficiently small $T$ (then, after having proved (iv), we can bootstrap the argument and achieve arbitrarily large time horizon $T$ ).

On the one hand, we proceed in a way analogous to the one followed to obtain (3.4) (recall that now we have $\chi=c_{m}=1$ ). Namely, we combine the equations on $v_{i, e}^{h, n}$ (with test function $u_{i, e}^{h, n}$ ) and on $w^{n, h}$ (with test function $(C / A) w^{h, n}$ ), use the 
convexity inequality in the place of chain rule in time, and we end up with

$$
\begin{aligned}
\int_{0}^{s} \int_{\Omega} & \left(\left(v^{h}\right)^{2} \mathcal{B}\left(\tilde{v}^{h}\right)-A_{1}\left(v^{h}\right)^{2}+\frac{B C}{A}\left(w^{h}\right)^{2}\right. \\
& \left.+\left(\mathbf{M}_{e}\left(\boldsymbol{x}, \mathbf{D} \boldsymbol{u}^{h}\right) \nabla v_{e}^{h}\right) \cdot \nabla v_{e}^{h}+\left(\mathbf{M}_{i}\left(\boldsymbol{x}, \mathbf{D} \boldsymbol{u}^{h}\right) \nabla v_{i}^{h}\right) \cdot \nabla v_{i}^{h}\right)+\int_{\Omega} \frac{1}{2}\left(v^{h}\right)^{2}(s, \cdot) \\
\leq & \int_{\Omega} \frac{1}{2}\left(v_{0}^{h}\right)^{2}+\int_{0}^{s} \int_{\Omega} I_{\text {app }}^{i, h} v_{i}^{h}-I_{\text {app }}^{e, h} v_{e}^{h},
\end{aligned}
$$

for every $s<T$. The we integrate in $s \in[0, T]$ and we assume $2 A_{1} T \leq 1$; we use the Fubini theorem to simplify the double time integral, which brings the factor $(T-t)$ under the integrals. Using the strong convergence of $v^{h, 0}, w^{h}$ and $\mathbf{D} \boldsymbol{u}^{h}$ in $L^{2}(\Omega), L^{2}\left(\Omega_{T}\right)$ and $L^{2}\left(\Omega_{T}\right)^{3}$, respectively, using the weak $L^{2}\left(\Omega_{T}\right)$ convergence of $v^{h}$, of $\sqrt{T-t} v^{h}$, of $\sqrt{T-t}\left(\mathbf{M}_{i, e}\left(\boldsymbol{x}, \mathbf{D} \boldsymbol{u}^{h}\right)\right)^{1 / 2} \nabla v_{i, e}^{h}$ and the lower semi-continuity of the $L^{2}$-norm with respect to weak convergence, at the limit $h \rightarrow 0$ (for the selected subsequence) we find

$$
\begin{aligned}
\limsup _{h \rightarrow 0} & \int_{0}^{T} \int_{\Omega}(T-t)\left(v^{h}\right)^{2} \mathcal{B}\left(\tilde{v}^{h}\right)+\int_{0}^{T} \int_{\Omega}\left(\left(\frac{1}{2}-(T-t) A_{1}\right) v^{2}+\frac{B C}{A} w^{2}\right) \\
& +\int_{0}^{T} \int_{\Omega}\left((T-t)\left(\mathbf{M}_{e}(\boldsymbol{x}, \mathbf{D} \boldsymbol{u}) \nabla v_{e}\right) \cdot \nabla v_{e}+\left(\mathbf{M}_{i}(\boldsymbol{x}, \mathbf{D} \boldsymbol{u}) \nabla v_{i}\right) \cdot \nabla v_{i}\right) \\
\leq & \int_{\Omega} \frac{T}{2}\left(v_{0}\right)^{2}+\int_{0}^{T} \int_{\Omega}(T-t)\left(I_{\mathrm{app}}^{i} v_{i}-I_{\mathrm{app}}^{e} v_{e}\right) .
\end{aligned}
$$

On the other hand, the following limit equations, as $h \rightarrow 0$, are easily obtained as $h \rightarrow 0$ :

$$
\begin{aligned}
\partial_{t} v-\nabla \cdot\left(\mathbf{M}_{i}(\boldsymbol{x}, \mathbf{D} \boldsymbol{u}) \nabla v_{i}\right)+\Psi-A_{1} v+C w & =I_{\text {app }}^{i} \quad \text { in } \Omega_{T}, \\
\partial_{t} v+\nabla \cdot\left(\mathbf{M}_{e}(\boldsymbol{x}, \mathbf{D} \boldsymbol{u}) \nabla v_{e}\right)+\Psi-A_{1} v+C w & =I_{\text {app }}^{e} \quad \text { in } \Omega_{T}, \\
v & =v_{i}-v_{e} \quad \text { in } \Omega_{T}, \\
\partial_{t} w-H(v, w) & =0 \quad \text { in } \Omega_{T} .
\end{aligned}
$$

In order to combine these equations in the same way as what we have done at the discrete level, we recall that $v \in L^{4}\left(\Omega_{T}\right)$ and $\Psi \in\left(L^{4}\left(\Omega_{T}\right)\right)^{\prime}$, so that Lemma 2.3 of Ref. 5 (cf. Remark 2.2 after Definition 2.1) can be used in order to give sense to multiplication of $(5.8),(5.9)$ by $v_{i}, v_{e}$, respectively. We obtain, integrating in $t \in[0, s]$ then in $s \in[0, T]$ the following relation:

$$
\begin{aligned}
\int_{0}^{T} \int_{\Omega} & (T-t) v \Psi+\int_{0}^{T} \int_{\Omega}\left(\left(\frac{1}{2}-(T-t) A_{1}\right) v^{2}+\frac{B C}{A} w^{2}\right) \\
& +\int_{0}^{T} \int_{\Omega}\left((T-t)\left(\mathbf{M}_{e}(\boldsymbol{x}, \mathbf{D} \boldsymbol{u}) \nabla v_{e}\right) \cdot \nabla v_{e}+\left(\mathbf{M}_{i}(\boldsymbol{x}, \mathbf{D} \boldsymbol{u}) \nabla v_{i}\right) \cdot \nabla v_{i}\right) \\
= & \int_{\Omega} \frac{T}{2}\left(v_{0}\right)^{2}+\int_{0}^{T} \int_{\Omega}(T-t)\left(I_{\mathrm{app}}^{i} v_{i}-I_{\mathrm{app}}^{e} v_{e}\right) .
\end{aligned}
$$

By comparison of (5.12) and (5.7), we deduce the claim (5.6). 
Now, using as in the proof of (ii) an increasing family of functions $\left(\mathcal{B}_{n}\right)_{n}$ approximating $\mathcal{B}$, we find for all $n$,

$$
\begin{aligned}
& \iint_{\Omega_{T}}(T-t) \int_{\mathbb{R}} \lambda^{2} \mathcal{B}_{n}(\lambda) d \nu_{(t, \boldsymbol{x})}(\lambda) d t d \boldsymbol{x} \\
& \quad=\lim _{h \rightarrow 0} \iint_{\Omega_{T}}(T-t)\left(v^{h}\right)^{2} \mathcal{B}_{n}\left(\tilde{v}^{h}\right) \leq \limsup _{h \rightarrow 0} \iint_{\Omega_{T}}(T-t)\left(v^{h}\right)^{2} \mathcal{B}\left(\tilde{v}^{h}\right) \\
& \quad \leq \iint_{\Omega_{T}}(T-t) v \Psi .
\end{aligned}
$$

Then with the monotone convergence theorem and representation of $\Psi$ and $v$ proved in (i), we find

$$
\begin{aligned}
& \iint_{\Omega_{T}}(T-t) \int_{\mathbb{R}} \lambda^{2} \mathcal{B}_{n}(\lambda) d \nu_{(t, \boldsymbol{x})}(\lambda) d t d \boldsymbol{x} \\
& \quad \leq \iint_{\Omega_{T}}(T-t)\left(\int_{\mathbb{R}} \lambda d \nu_{(t, \boldsymbol{x})}(\lambda)\right)\left(\int_{\mathbb{R}} \lambda \mathcal{B}(\lambda) d \nu_{(t, \boldsymbol{x})}(\lambda)\right) d t d \boldsymbol{x} .
\end{aligned}
$$

Then, rearranging the terms as in Ref. 21, one finds

$$
\int_{0}^{T} \int_{\Omega}(T-t) \int_{\mathbb{R}} \int_{\mathbb{R}}(\lambda-\mu)(\lambda \mathcal{B}(\lambda)-\mu \mathcal{B}(\mu)) d \nu_{(t, \boldsymbol{x})}(\lambda) d \nu_{(t, \boldsymbol{x})}(\mu) d t d \boldsymbol{x} \leq 0 .
$$

Now the claim of (iii) follows by monotonicity of the map $\lambda \mapsto \lambda \mathcal{B}(\lambda)$.

(iv) Starting from the "div-curl" relation proved in (iii), one deduces the claim (iv) from the general properties of Young measures (see Ref. 21).

Property (iv) of the previous lemma, along with Eqs. (5.8), (5.9) concludes the passage to the limit in the scheme. It remains to observe that, if the data of the problem allow for existence of a bounded-gradient solution defined in Sec. 4, the extraction of a subsequence is bypassed using the classical argument (the unique solution is the unique accumulation point), and in this case the finite element method converges to the unique solution of the system. A priori error estimates can also be obtained in this case, with arguments similar to those of Sec. 4 , but we restrict ourselves to assess the experimental convergence of the scheme in Sec. 5.3.

\subsection{Numerical tests}

We now illustrate the performance of the coupled finite element method. The linear systems arising after Newton linearization of the coupling and reaction terms and full discretization of the problem are solved with the GMRES method with a tolerance of $\hat{\epsilon}_{\text {tol }}=10^{-7}$. We set a tolerance of $\tilde{\epsilon}_{\text {tol }}=10^{-8}$ for the $L^{2}$-norm of the residual of Newton iterates. All the simulations in this section have been performed on four cluster nodes with two Intel Xeon processors (quad core, 8MB cache, 2.66 Ghz CPU) each. 
First, we assess the convergence rate of the finite element approximation of deformation, pressure, and electric potentials proposed in Sec. 5.1. We focus on the spatial convergence of the numerical scheme, and so we analyze the experimental convergence of the following steady state counterpart of (5.1):

$$
\begin{aligned}
&\left(\boldsymbol{\sigma}\left(\boldsymbol{x}, \gamma^{h}\right) \mathbf{D} \boldsymbol{u}^{h}, \mathbf{D} \boldsymbol{\psi}^{h}\right)_{\Omega}-\left(p^{h}, \nabla \cdot \boldsymbol{\psi}^{h}\right)_{\Omega}=\left(\boldsymbol{f}^{h}, \boldsymbol{\psi}^{h}\right)_{\Omega}+\left(\alpha \boldsymbol{u}^{h}, \boldsymbol{\psi}^{h}\right)_{\partial \Omega}, \\
&-\left(\nabla \cdot \boldsymbol{u}^{h}, q^{h}\right)_{\Omega}=0, \\
&\left(\mathbf{M}_{i}\left(\boldsymbol{x}, \mathbf{D} \boldsymbol{u}^{h}\right) \nabla v_{i}^{h}, \nabla \phi_{i}^{h}\right)_{\Omega}+\left(I_{\mathrm{ion}}\left(v^{h}, w^{h}\right), \phi_{i}^{h}\right)_{\Omega}=I_{\mathrm{app}}^{i}, \\
&-\left(\mathbf{M}_{e}\left(\boldsymbol{x}, \mathbf{D} \boldsymbol{u}^{h}\right) \nabla v_{e}^{h}, \nabla \phi_{e}^{h}\right)_{\Omega}+\left(I_{\mathrm{ion}}\left(v^{h}, w^{h}\right), \phi_{e}^{h}\right)_{\Omega}=I_{\mathrm{app}}^{e},
\end{aligned}
$$

for all $\psi^{h} \in \mathcal{V}^{h}, q^{h}, \phi_{h}^{i}, \phi_{h}^{e} \in V_{h}$. Notice that in this specific case, $w^{h}, \gamma^{h}$ can be obtained by post-processing from $v^{h}$ using the equilibrium equations $H\left(v^{h}, w^{h}\right)=$ $G\left(\gamma^{h}, w^{h}\right)=0$. We use model parameters as specified in Table 1 , and subsequently $I_{\text {ion }}=I_{\text {ion }}\left(v^{h}\right)=\left(v^{h}\right)^{3}+v^{h}$. Let us consider the slab of tissue represented by the unit square $\Omega=(0,1)^{2}$, where Robin data (with coefficient $\alpha=\mu$ ) are imposed on the whole boundary and zero-flux conditions are assumed for the electric potentials. Fibers and sheet directions are fixed as $\boldsymbol{d}_{l}=(0,1)^{T}$ and $\boldsymbol{d}_{t}=(1,0)^{T}$, respectively. An exact solution of the problem is given by the following smooth functions:

$$
\begin{aligned}
& \boldsymbol{u}=\left(\begin{array}{c}
\frac{x}{10}+\sqrt{1.001} y \\
-\frac{y}{10}-\sqrt{1.001} x
\end{array}\right), \quad p=\frac{\mu}{10} x^{2}(x+\sqrt{1.001} y), \\
& v=-\gamma_{R} \exp \left(-4[y-1 / 2]^{2}\right), \quad v_{i}=v+x y, \quad v_{e}=x y,
\end{aligned}
$$

which satisfy both incompressibility constraints $\operatorname{det} \mathbf{D} \boldsymbol{u}=1$ and $\nabla \cdot \boldsymbol{u}=0$. The forcing term $f$ and the applied currents $I_{\text {app }}^{i . e}$ are manufactured according to these exact solutions. The slab is partitioned into successively refined meshes with $2^{n}+1$, $n=0,1, \ldots, 8$ vertices on each side of the domain and we compute errors and observed convergence rates defined as

$$
\begin{aligned}
e(\boldsymbol{u}): & :=\left\|\boldsymbol{u}-\boldsymbol{u}_{h}\right\|_{H^{1}(\Omega)}, \quad e(s):=\left\|s-s_{h}\right\|_{H^{1}(\Omega)}, \\
r(\boldsymbol{u}): & =\frac{\log (e(\boldsymbol{u}) / \hat{e}(\boldsymbol{u}))}{\log (h / \hat{h})}, \quad r(s):=\frac{\log (e(s) / \hat{e}(s))}{\log (h / \hat{h})},
\end{aligned}
$$

where $s$ denotes an electric potential or pressure, and $e$ and $\hat{e}$ denote errors computed on two consecutive meshes of sizes $h$ and $\hat{h}$, respectively. Table 2 reports the convergence history for the piecewise quadratic approximation of deformations and piecewise linear approximation of all remaining scalar fields, where we can observe optimal convergence rates in all cases. The approximate solutions are displayed in Fig. 1.

Next, we turn to the simulation of the transient linearized coupled cardiac electromechanical problem (2.12)-(2.20). A human heart geometry and fiber directions obtained from CT scan data ${ }^{41}$ have been smoothed, rescaled and meshed using the 


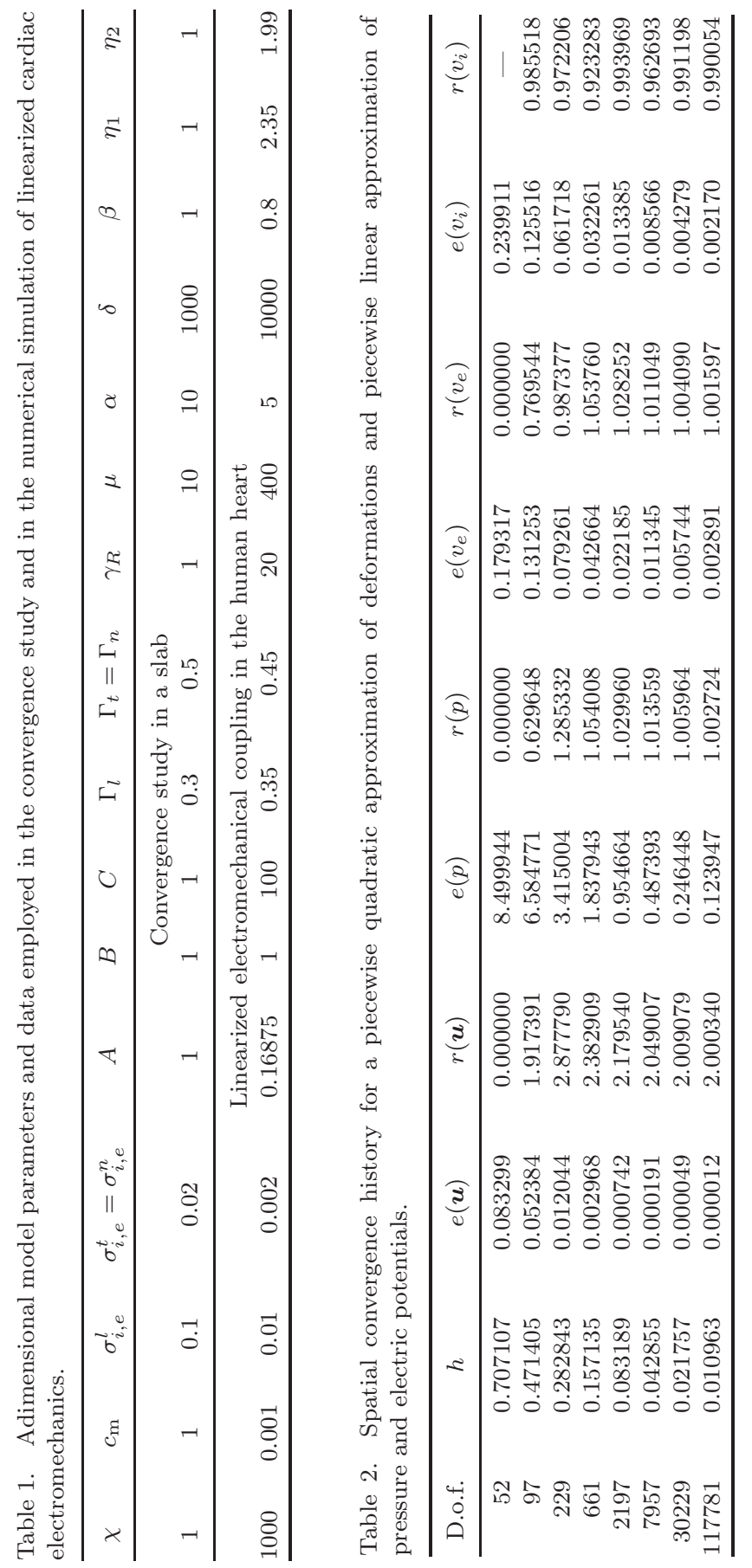



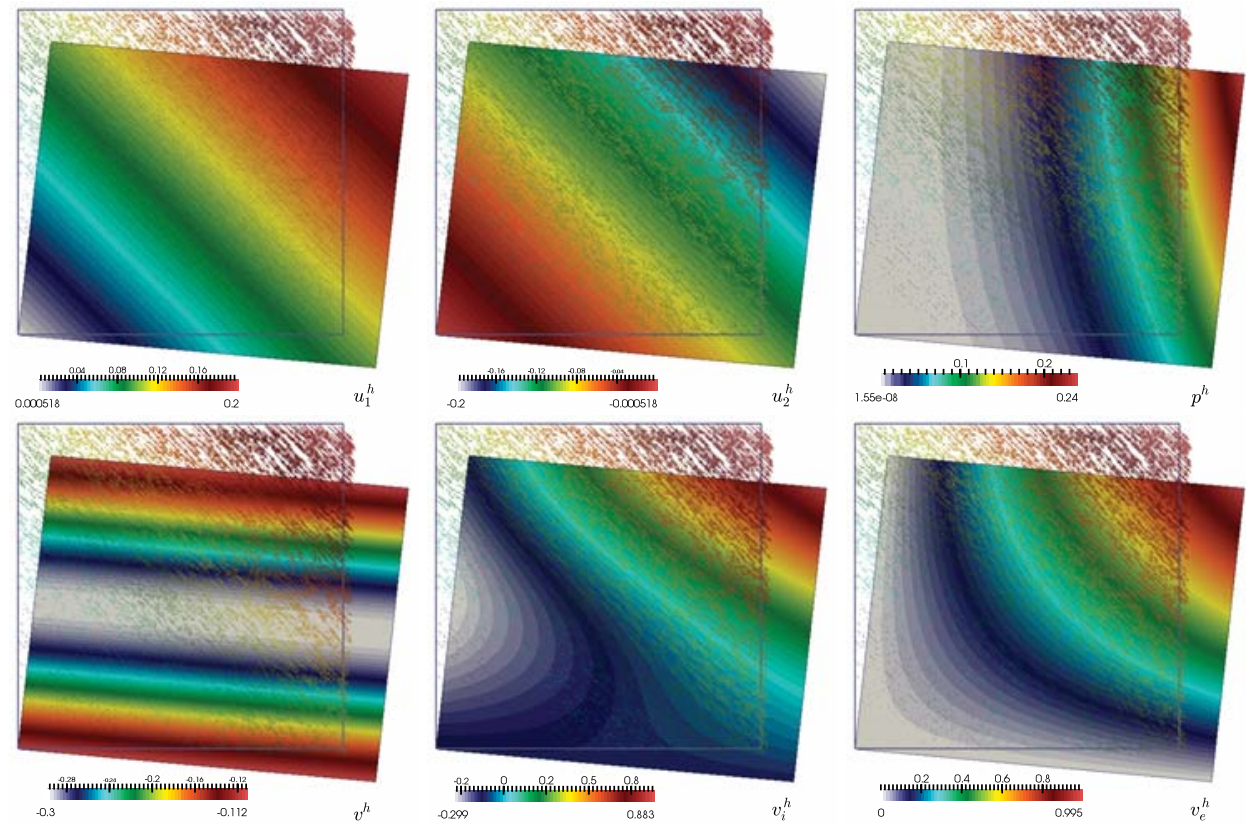

Fig. 1. Approximate solutions of the convergence test displayed on the deformed configuration (deformation components, pressure, transmembrane potential, intra- and extra-cellular potential).
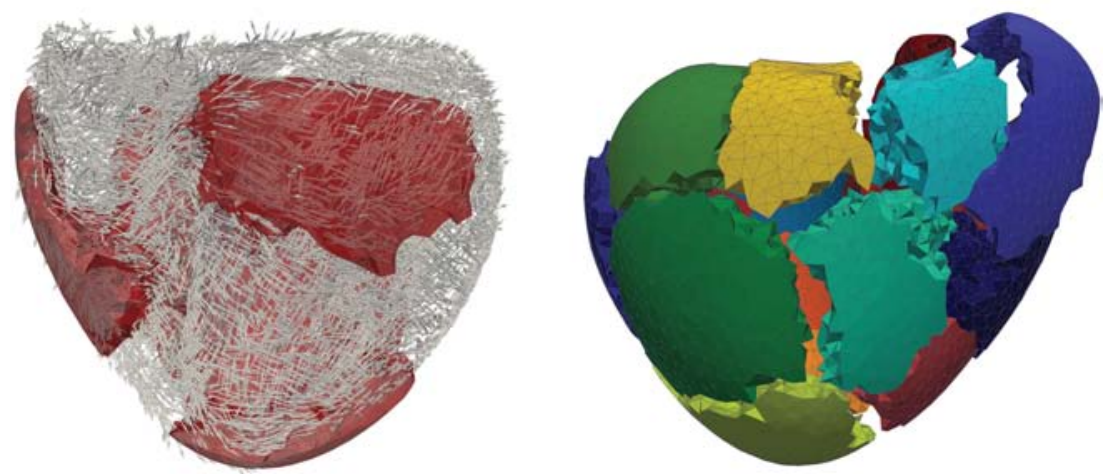

Fig. 2. Fibers distribution with segmented biventricular geometry (left) and exploded view of the computational mesh partitioned into 16 nonoverlapping subdomains, each color representing a different subdomain (right).

VMTK library (http://www.vmtk.org) (see Fig. 2). The resulting idealized biventricular mesh consists of 94590 four-node elements and 23210 vertices. The transmembrane potential is initially at rest $v=-84 \mathrm{mV}$ and the excitation propagation is initiated with a stimulus of magnitude $100 \mathrm{mV}$ applied on the septum and apical zone of left and right ventricles at time $t=10$. The forcing term for the elasticity 
equation is zero $\boldsymbol{f}=\mathbf{0}$. Other model parameters are set as specified in the bottom row of Table 1 . A time step $\Delta t=0.01$ is employed and an average overall CPU time spent per Newton step is $12.5 \mathrm{~s}$, for an average iteration count of nine steps to achieve the desired convergence. We simulate one full heartbeat and report in
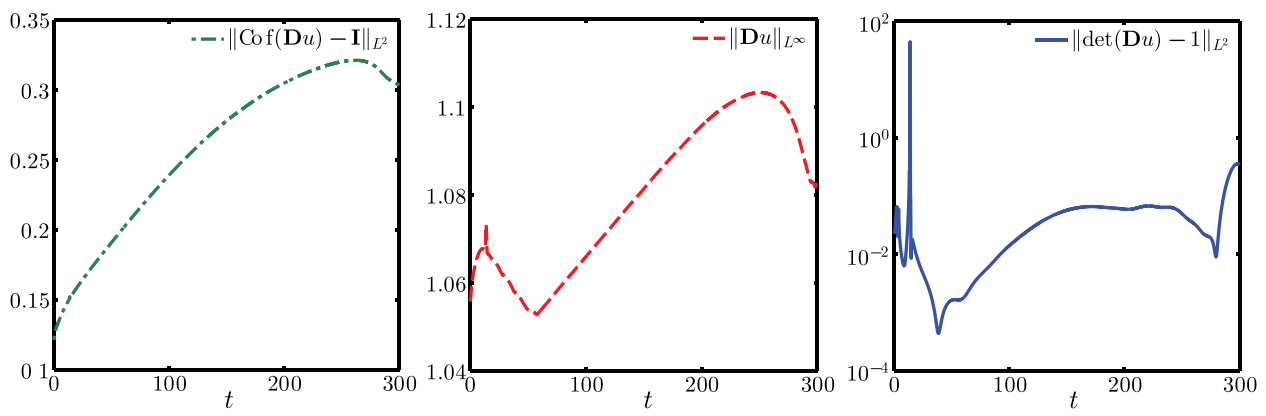

Fig. 3. Time evolution over half a heartbeat of the numerical residuals induced by the linearization of the elasticity problem.
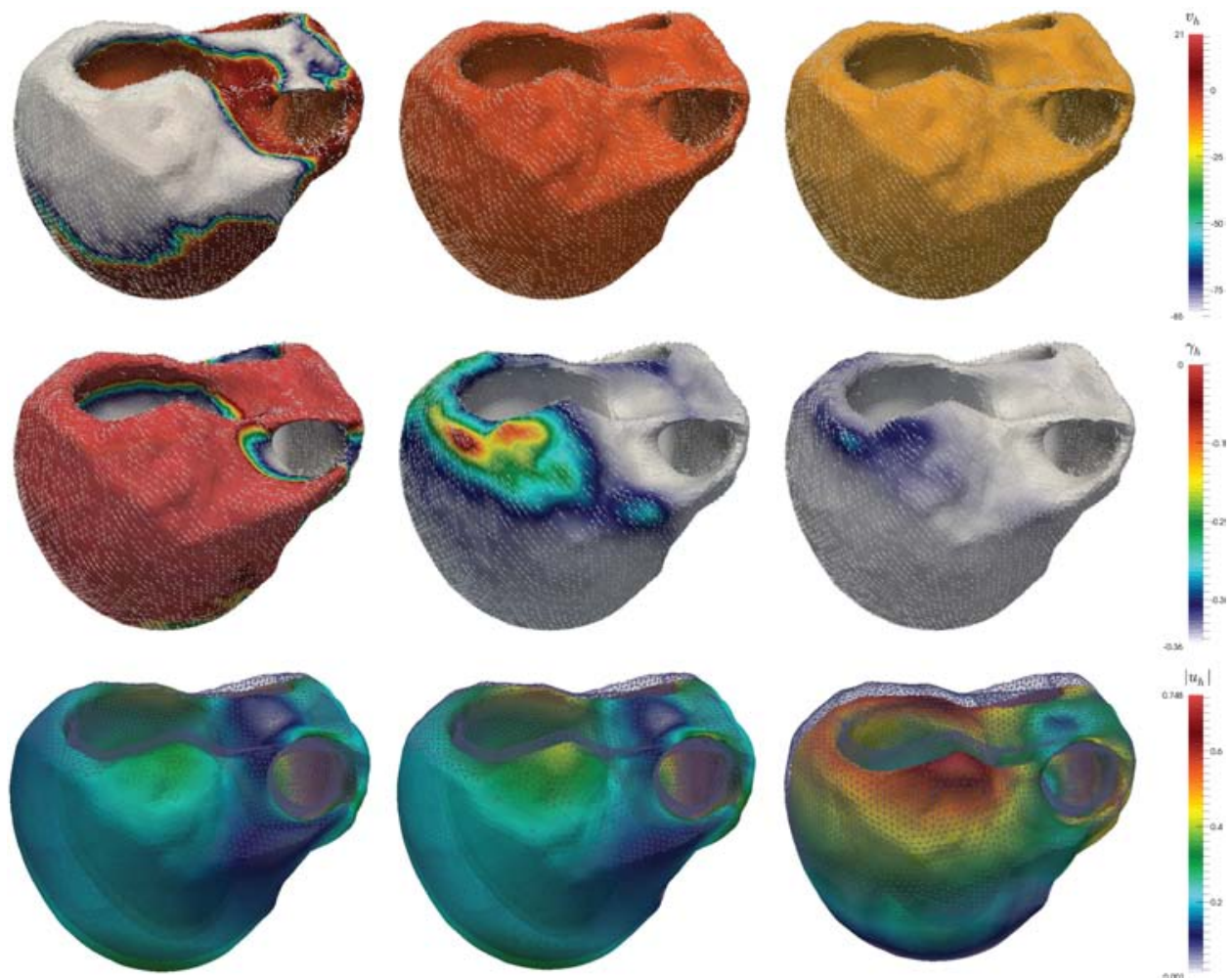

Fig. 4. Transmembrane potential (top), activation function (middle) and tissue deformation (bottom) at time instants $t=100,150,300$ (from left to right). 
Fig. 4 snapshots of the spatio-temporal evolution of action potential and tissue deformation. We have applied several model simplifications for sake of the analysis, including phenomenological FitzHugh-Nagumo membrane kinetics, linearized mechanical response, and stretch-independent activation. Evidently, some features of the cardiac function will be difficult to recover within this framework, such as accurate representations of stresses at high strains. In addition, we can observe that the following residuals

$$
\|\operatorname{Cof}(\mathbf{D} \boldsymbol{u})-\mathbf{I}\|_{L^{2}(\Omega)}, \quad\|\mathbf{D} \boldsymbol{u}\|_{L^{\infty}(\Omega)}, \quad\|\operatorname{det}(\mathbf{D} \boldsymbol{u})-1\|_{L^{2}(\Omega)}
$$

are non-negligible (see the dynamics depicted in Fig. 3, where the spike in the middle and right plots is due to the electrical stimulus applied at $t=10$ ). In the fully nonlinear case, performing Newton or Picard iterations (also for the mechanical problem) up to a fixed tolerance rapidly decreases the first residual, associated with the nonlinear stress. Nevertheless, the order of magnitude of the second and third residuals remain unchanged, suggesting that total strains and incompressibility are well resolved even in the linear case. Moreover, from Fig. 4 we readily observe some other key features such as the desired delay of the activation $\gamma$ with respect to the front of the transmembrane potential, and the subsequent contraction of the muscle.

\section{Concluding Remarks}

We have introduced a mathematical model for the study of cardiac electromechanical interactions written in fully Lagrangian form, featuring a linearized description of the passive elastostatics of cardiac tissue, a linearized incompressibility constraint, and a truncated approximation of the inverse Cauchy Green tensor appearing in the updated conductivity term of the bidomain equations. The existence of weak solutions to the coupled problem has been established by convergence of Galerkin approximations and regularization. The concept of bounded-gradient weak solutions has been instrumental in the derivation of uniqueness of weak solutions in the natural norms, and we have presented some ideas on the analysis of regularity. A finite element method has been introduced for the approximation of the electromechanical system, and we have provided sketches for its convergence proof. The experimental convergence of the numerical scheme and the applicability of the simplified model in the study of cardiac electromechanics have finally addressed via two numerical examples.

We have taken the FitzHugh-Nagumo kinetics as the simplest example, but the extension of the present theoretical framework to other linear phenomenological ionic models such as the Aliev-Panfilov, ${ }^{1}$ Rogers-McCulloch, ${ }^{38}$ or MitchellSchaeffer ${ }^{28}$ models is straightforward, provided the evolution of the activation parameter $\gamma$ is also governed by a linear ODE. However, more involved (typically physiologically-based) nonlinear ionic models could not be addressed by the same mathematical techniques put forward in our work. First, a priori estimates for the 
transmembrane potential need to be re-derived from the modified ODE system governing the ionic activity (cf. the existence results of Ref. 49 for Luo-Rudy I kinetics, see also Ref. 11 for a regularized Mitchell-Schaeffer model). As a consequence of such estimates, a convenient duality is established between the functional spaces for the transmembrane potential and for the ionic current. These features are natural, because they are related to stability and to the cyclic evolution of solutions to the pure ODE model neglecting spatial propagation effects. However, the simple space-translation argument that we employed in Sec. 3.2 is no longer appropriate if some ODEs governing reactions, including the activation field $\gamma$, are nonlinear (which e.g. would be the case for the Luo-Rudy models). Alternative approaches may involve theoretical tools for the investigation of stability of solutions to ODEs (cf. Ref. 49), or differentiation of the ODEs with respect to the space variable $\boldsymbol{x}$. While both of these techniques might be helpful for studying existence (using, e.g. the uniformly parabolic "viscous" approximation of ODEs of finite volume approximations, cf. Ref. 5), the structure of Galerkin and finite element approximations studied here jeopardizes these strategies of proof. Indeed, except for the linear case, the equations obtained from finite element approximation of ODEs are not satisfied pointwise with respect to the space parameter $\boldsymbol{x}$, but only in some averaged-in- $\boldsymbol{x}$ sense.

Although we have recently tested numerically more complex and more realistic formulations for cardiac electromechanics, ${ }^{23,33,39,42}$ the model simplifications applied in the present study were mainly driven by the need of addressing solvability and regularity questions often overlooked in the literature. In addition, some extensions towards physiological relevance can be readily applied without changing the core of the theoretical tools employed herein. For instance, the role of anisotropy is not substantial in our present analysis. In fact, once the activation is applied to the passive mechanics via the active strain formalism, the stress tensors (first Piola-Kirchhoff and Cauchy's) adopt an anisotropic structure even if their passive forms are isotropic. Moreover, since the elastostatics are linearized, further generalizations to e.g. exponential laws with several fiber families, as e.g. the Holzapfel-Ogden model, ${ }^{20}$ could be readily incorporated in the present framework.

For the fully nonlinear case the situation is more delicate, since coercivity of the stress (which in the case of finite elasticity is guaranteed by polyconvexity of the strain energy function ${ }^{6}$ ) will depend on the specific material law each case would need to be analyzed separately. In this regard, the active strain approach represents a major advantage, since the activation via multiplicative splitting of the deformation gradient tensor does not modify the stability properties originally featured by the passive mechanical law. ${ }^{4,33}$

In conclusion, deeper theoretical insight and numerical experiments are needed to assess the quality of more realistic cardiac electrophysiological models with nonlinear elastic behavior and nonlinear ionic evolution. 


\section{Acknowledgments}

This work was initiated during visits of M. Bendahmane to the EPF Lausanne and the Université de Franche-Comté, and he is grateful for the hospitality. A.Q. and R.R.-B. acknowledge the financial support by the European Research Council through the advanced Grant "MATHCARD, Mathematical Modelling and Simulation of the Cardiovascular System", project ERC-2008-AdG 227058.

\section{References}

1. R. Aliev and A. V. Panfilov, A simple two-variable model of cardiac excitation, Chaos, Solitons Fractals 7 (1996) 293-301.

2. H. W. Alt and S. Luckhaus, Quasilinear elliptic-parabolic differential equations, Math. Z. 183 (1983) 311-342.

3. D. Ambrosi, G. Arioli, F. Nobile and A. Quarteroni, Electromechanical coupling in cardiac dynamics: The active strain approach, SIAM J. Appl. Math. 71 (2011) 605621.

4. D. Ambrosi and S. Pezzuto, Active stress versus active strain in mechanobiology: Constitutive issues, J. Elasticity 107 (2012) 199-212.

5. B. Andreianov, M. Bendahmane, K. H. Karlsen and C. Pierre, Convergence of discrete duality finite volume schemes for the cardiac bidomain model, Netw. Heterog. Media 6 (2011) 195-240.

6. J. M. Ball, Convexity conditions and existence theorems in nonlinear elasticity, Arch. Rational Mech. Anal. 63 (1976-1977) 337-403.

7. D. Baroli, A. Quarteroni and R. Ruiz-Baier, Convergence of a stabilized discontinuous Galerkin method for incompressible nonlinear elasticity, Adv. Comput. Math. 39 (2013) 425-443.

8. M. Bendahmane and K. H. Karlsen, Analysis of a class of degenerate reactiondiffusion systems and the bidomain model of cardiac tissue, Netw. Heterog. Media 1 (2006) 185-218.

9. D. M. Bers, Cardiac excitation-contraction coupling, Nature 415 (2002) 198-205.

10. R. M. Bordas, K. Gillow, D. Gavaghan, B. Rodriguez and D. Kay, A bidomain model of the ventricular specialized conduction system of the heart, SIAM J. Appl. Math. 72 (2012) 1618-1643.

11. M. Boulakia, M. A. Fernández, J.-F. Gerbeau and N. Zemzemi, A coupled system of PDEs and ODEs arising in electrocardiograms modeling, Appl. Math. Res. Express AMRX 2 (2008), abn002, 28 pp.

12. Y. Bourgault, Y. Coudière and C. Pierre, Existence and uniqueness of the solution for the bidomain model used in cardiac electrophysiology, Nonlinear Anal.: Real World Appl. 10 (2009) 458-482.

13. C. Cherubini, S. Filippi, P. Nardinocchi and L. Teresi, An electromechanical model of cardiac tissue: Constitutive issues and electrophysiological effects, Progr. Biophys. Molec. Biol. 97 (2008) 562-573.

14. P. G. Ciarlet, Mathematical Elasticity, Vol I: Three-Dimensional Elasticity (NorthHolland, 1978).

15. P. Colli Franzone and G. Savaré, Degenerate evolution systems modeling the cardiac electric field at micro- and macroscopic level, in Evolution Equations, Semigroups and Functional Analysis, Progress in Nonlinear Differential Equations Application, Vol. 50 (Birkhäuser, 2002), pp. 49-78. 
16. M. Ethier and Y. Bourgault, Semi-implicit time-discretization schemes for the bidomain model, SIAM J. Numer. Anal. 46 (2008) 2443-2468.

17. M. Fernández and N. Zemzemi, Decoupled time-marching schemes in computational cardiac electrophysiology and ECG numerical simulation, Math. Biosci. 226 (2010) $58-75$.

18. R. FitzHugh, Impulses and physiological states in theoretical models of nerve membrane, Biophys. J. 1 (1961) 445-465.

19. S. Göktepe and E. Kuhl, Electromechanics of the heart: A unified approach to the strongly coupled excitation-contraction problem, Comput. Mech. 45 (2010) 227-243.

20. G. A. Holzapfel and R. W. Ogden, Constitutive modelling of passive myocardium: A structurally-based framework for material characterization, Philos. Trans. Roy. Soc. A 367 (2009) 3445-3475.

21. N. Hungerbühler, Quasi-linear parabolic systems in divergence form with weak monotonicity, Duke Math. J. 107 (2001) 497-520.

22. P. Krejčí, J. Sainte-Marie, M. Sorine and J. M. Urquiza, Solutions to muscle fiber equations and their long time behaviour, Nonlinear Anal. Real World Appl. 7 (2006) $535-558$.

23. A. Laadhari, R. Ruiz-Baier and A. Quarteroni, Fully Eulerian finite element approximation of a fluid-structure interaction problem in cardiac cells, Int. J. Numer. Methods Engrg. 96 (2013) 712-738.

24. P. Lafortune, R. Arís, M. Vázquez and G. Houzeaux, Coupled electromechanical model of the heart: Parallel finite element formulation, Int. J. Numer. Methods Biomed. Engrg. 28 (2012) 72-86.

25. G. M. Lieberman, Boundary regularity for solutions of degenerate parabolic equations, Nonlinear Anal. 14 (1990) 501-524.

26. J.-L. Lions, Quelques Méthodes de Résolution des Problèmes aux Limites Non Linéaires (Dunod, 1969).

27. H. Matano and Y. Mori, Global existence and uniqueness of a three-dimensional model of cellular electrophysiology, Discrete Contin. Dynam. Syst. 29 (2011) 1573-1636.

28. C. C. Mitchell and D. G. Schaeffer, A two-current model for the dynamics of cardiac membrane, Bull. Math. Biol. 65 (2003) 767-793.

29. J. S. Nagumo, S. Arimoto and S. Yoshizawa, An active pulse transmission line stimulating nerve axon, Proc. Inst. Radio Engrg. 50 (1962) 2061-2071.

30. P. Nardinocchi and L. Teresi, On the active response of soft living tissues, J. Elasticity 88 (2007) 27-39.

31. M. P. Nash and P. J. Hunter, Computational mechanics of the heart. From tissue structure to ventricular function, J. Elasticity 61 (2000) 113-141.

32. M. P. Nash and A. V. Panfilov, Electromechanical model of excitable tissue to study re-entrant cardiac arrhythmias, Progr. Biophys. Molec. Biol. 85 (2004) 501-522.

33. F. Nobile, A. Quarteroni and R. Ruiz-Baier, An active strain electromechanical model for cardiac tissue, Int. J. Numer. Meth. Biomed. Engrg. 28 (2012) 52-71.

34. D. A. Nordsletten, S. A. Niederer, M. P. Nash, P. J. Hunter and N. P. Smith, Coupling multi-physics models to cardiac mechanics, Progr. Biophys. Molec. Biol. 104 (2011) 77-88.

35. P. Pathmanathan, S. J. Chapman, D. J. Gavaghan and J. P. Whiteley, Cardiac electromechanics: The effect of contraction model on the mathematical problem and accuracy of the numerical scheme, Quart. J. Mech. Appl. Math. 63 (2010) 375-399.

36. P. Pathmanathan, C. Ortner and D. Kay, Existence of solutions of partially degenerate visco-elastic problems, and applications to modelling muscular contraction and cardiac electro-mechanical activity, submitted. 
37. A. Quarteroni and A. Valli, Numerical Approximation of Partial Differential Equations, Springer Series in Computational Mathematics, Vol. 23 (Springer, 1994).

38. J. M. Rogers and A. D. McCulloch, A collocation-Galerkin finite element model of cardiac action potential propagation, IEEE Trans. Biomed. Engrg. 41 (1994) 743-757.

39. S. Rossi, T. Lassila, R. Ruiz-Baier, A. Sequeira and A. Quarteroni, Thermodynamically consistent orthotropic activation model capturing ventricular systolic wall thickening in cardiac electromechanics, Eur. J. Mech. A Solids 48 (2014) 129-142.

40. S. Rossi, R. Ruiz-Baier, L. F. Pavarino and A. Quarteroni, Orthotropic active strain models for the numerical simulation of cardiac biomechanics, Int. J. Numer. Meth. Biomed. Engrg. 28 (2012) 761-788.

41. O. Rousseau, Geometrical modeling of the heart, Ph.D. thesis, Université d'Ottawa (2010).

42. R. Ruiz-Baier, D. Ambrosi, S. Pezzuto, S. Rossi and A. Quarteroni, Activation models for the numerical simulation of cardiac electromechanical interactions, in Computer Models in Biomechanics: From Nano to Macro, eds. G. A. Holzapfel and E. Kuhl (Springer, 2013), pp. 189-201.

43. R. Ruiz-Baier, A. Gizzi, S. Rossi, C. Cherubini, A. Laadhari, S. Filippi and A. Quarteroni, Mathematical modeling of active contraction in isolated cardiomyocytes, Math. Med. Biol. 31 (2014) 259-283.

44. S. Sanfelici, Convergence of the Galerkin approximation of a degenerate evolution problem in electrocardiology, Numer. Methods Partial Differential Equations 18 (2002) 218-240.

45. J. Sundnes, G. T. Lines, X. Cai, B. F. Nielsen, K.-A. Mardal and A. Tveito, Computing the Electrical Activity in the Heart, Monographs in Computational Science and Engineering, Vol. 1 (Springer, 2006).

46. J. Sundnes, S. Wall, H. Osnes, T. Thorvaldsen and A. D. McCulloch, Improved discretisation and linearisation of active tension in strongly coupled cardiac electromechanics simulations, Comput. Meth. Biomech. Biomed. Engrg. 17 (2014) 604-615.

47. N. A. Trayanova, Whole-heart modeling: Applications to cardiac electrophysiology and electromechanics, Circ. Res. 108 (2011) 113-128.

48. L. Tung, A bidomain model for describing ischemic myocardial D-C potentials, Ph.D. thesis, MIT (1978).

49. M. Veneroni, Reaction-diffusion systems for the macroscopic bidomain model of the cardiac electric field, Nonlinear Anal. Real World Appl. 10 (2009) 849-868. 